UNITU-THEP-08/1996

nucl-th/9609012

\title{
Baryons as Hybrids of Solitons and Three Quark Bound States
}

\author{
U. Zückert, R. Alkofer, H. Weigel, and H. Reinhardt \\ Institute for Theoretical Physics, Tübingen University \\ Auf der Morgenstelle 14, D-72076 Tübingen, Germany
}

(October 4, 2018)

\begin{abstract}
A hybrid model for baryons based on a dynamical interplay between relativistic three-quark bound states and soliton configurations of mesons is constructed. The Bethe-Salpeter equation for diquarks and the Faddeev equation for diquark-quark bound states in the background of a soliton is solved. The results show that baryons are very much like hybrids containing both, solitonic meson clouds and three-quark correlations.
\end{abstract}

PACS: 12.39.Fe, 12.39.Ki, 12.40.Yx, 11.10.St, 11.80.Jy

Typeset using REVTEX 


\section{INTRODUCTION}

At present there exist two generic classes of models to describe baryons. On the one hand, there is the picture of baryons as chiral solitons. The soliton picture is based on considering QCD for an arbitrary number of color degrees of freedom, $N_{C}$. In the infinite color limit, $N_{C} \rightarrow \infty$, QCD reduces to an effective theory of infinitely many weakly interacting mesons [1]. Although this effective meson theory cannot be constructed explicitly, Witten conjectured that within this theory baryons emerge as solitons [2]. Based on this conjecture phenomenological effective meson theories have been developed which possess soliton solutions. The most prominent is perhaps the Skyrme model [3] [- In the limit of an infinite number of colors the soliton description is the only model for baryons. On the other hand, for a finite number of colors a baryon is customarily considered as a bound state of three valence quarks. Such valence quark models are motivated by high energy scattering experiments which have revealed a partonic substructure. Starting with these experimental facts many models, which are based on the valence quark picture, have been developed. These models include the non-relativistic quark models [6 8] and its relativistic extensions [9], parton models which are directly based on the scale invariance [10], bag models [11] and diquark-quark models [12,13].

The valence quark picture directly leads to the quantum numbers of a physical baryon whereas the soliton can only be interpreted as a baryon with good spin and flavor quantum numbers after collective quantization. Yet, baryons as solitons are conceptually better suited for the description of low energy properties because they straightforwardly embed the useful feature of chiral symmetry and its spontaneous breaking. In any event, despite their successes both pictures possess only limited ranges of applicability.

Since the advantages of both pictures are in some sense complementary a unification of the two approaches seems desirable. In principle, the chiral bag model [14, 15] represents such a combination since inside the bag it contains explicit quark degrees of freedom whereas a chiral soliton field surrounds the bag. As a consequence of the Chesire cat principle [16] experimental measurable quantities should not depend on the radius chosen for the bag. Explicit calculations show that the Chesire cat principle is not universally valid. In particular, the singlet axial matrix element depends strongly on the bag radius 17, 18. Therefore, a hybrid model is desirable which connects both classes of models in a dynamical fashion. In this context the Nambu-Jona-Lasinio (NJL) model [19] is for the moment unique. On the one hand, it possesses soliton solutions [20] of meson fields which themselves are obtained as bound antiquark-quark states [21]. On the other hand, within this model baryons may be described as three-quark bound states via the use of diquarks 2228 , while the meson fields are fixed to their vacuum expectation values. In particular this model is unique because with the help of path integral hadronization techniques [29] 
a consistent unification of both approaches is possible without any double counting of correlations.

Because of the enormous computational effort needed, the realization of a rigorous self-consistent solution is for the moment not feasible. Nevertheless, an approximate evaluation of this hybrid baryon can be accomplished within a four step procedure to be carried out in this paper: In the proceeding section the transformation of the NJL model with a pointlike interaction of color octet current into an effective theory of mesons, diquarks and baryons is described. For completeness we briefly repeat the first two steps in the beginning of the third section: First, we construct a static ground state solution in the absence of diquark and elementary baryon fields. Second, we solve the Bethe-Salpeter equation for a scalar diquark in the solitonic background field [30. In addition, we derive the Faddeev equation for arbitrary quark propagators. In section [V we discuss numerical results for the solutions of the Bethe-Salpeter equation as well as of the Faddeev equation. In section $\square$ we employ these results to define the hybrid model and discuss some static properties. We close with conclusions and a outlook in section VI. Some details of the calculation and a few lengthy formulas are left to appendices.

\section{HADRONIZATION OF THE NJL MODEL}

As stated in the introduction we consider a NJL model for two flavors

$$
\mathcal{L}_{N J L}=\bar{q}\left(i \not \partial-\hat{m}^{0}\right) q-\frac{1}{2} g j_{\mu}^{a} j_{a}^{\mu}
$$

with a pointlike interaction of color octet flavor singlet currents $j_{\mu}^{a}=\bar{q} \lambda_{C}^{a} \gamma_{\mu} q / 2$. Here $q$ denotes the quark spinors, $\hat{m}^{0}=\operatorname{diag}\left(m_{0}^{u}, m_{0}^{d}\right)$ the current quark mass matrix for two flavors and $\lambda_{C}^{a}\left(a=1, . ., N_{C}^{2}-1\right)$ are the generators of color $S U\left(N_{C}\right)$. The interaction part can be Fierz-rearranged to solely attractive channels

$$
\mathcal{L}_{\text {int }}=g_{1}\left(1-\frac{1}{N_{C}}\right)\left(\bar{q} \Lambda_{\alpha} q\right)\left(\bar{q} \Lambda^{\alpha} q\right)+\frac{g_{2}}{N_{C}}\left(\bar{q} \Gamma_{\alpha} q^{c}\right)\left(\bar{q}^{c} \Gamma^{\alpha} q\right)
$$

where $q^{c}=C \bar{q}^{T}$ denotes the charge conjugated Dirac spinor. Furthermore, we have defined the vertices

$$
\Lambda^{\alpha}=\mathbb{1}_{c} \frac{\tau^{a}}{2} \mathcal{O}^{\mathrm{a}}, \quad \Gamma^{\alpha}=\left(\frac{i \epsilon^{A}}{\sqrt{2}}\right)_{c} \frac{\tau^{a}}{2} \mathcal{O}^{\mathrm{a}} \quad \text { with } \quad \alpha=(A, a, \mathrm{a})
$$

for the quark-antiquark and the quark-quark interaction, respectively. $\mathcal{O}^{\mathrm{a}}$ corresponds to the set of Dirac matrices

$$
\mathcal{O}^{\mathrm{a}} \in\left\{\mathbb{1}, i \gamma_{5}, \frac{i \gamma^{\mu}}{\sqrt{2}}, \frac{i \gamma^{\mu} \gamma_{5}}{\sqrt{2}}\right\}
$$


and $\tau^{a}$ are isospin matrices. Because of the Fierz-transformation the coupling constants are restricted to $g_{1}=g_{2}=g$, which is necessary to preserve consistent $N_{C}$ counting. Note, that the diquark channel is suppressed by a factor $1 / N_{C}$ in comparison to the meson channel. Henceforth we will confine the discussion to the physical case $N_{C}=3$, unless explicit noted.

To convert the pure quark NJL model (2.1) into an effective hadron theory] [29] we introduce collective meson $\varphi^{\alpha}$ and baryon fields $B_{\alpha}^{\beta}$ and $\bar{B}_{\alpha}^{\beta}$ into the generating functional

$$
Z=\int \mathcal{D} q \mathcal{D} \bar{q} \exp \left(\int \mathcal{L}_{N J L}\right)
$$

via the identities

$$
\begin{aligned}
\mathbb{1} & =\int \mathcal{D} \chi_{\alpha} \delta\left(\chi_{\alpha}-\bar{q} \Lambda_{\alpha} q\right)=\int \mathcal{D} \chi_{\alpha} \mathcal{D} \varphi^{\alpha} \exp \left(i \int \varphi^{\alpha}\left(\chi_{\alpha}-\bar{q} \Lambda_{\alpha} q\right)\right) \\
\mathbb{1} & =\int \mathcal{D} \bar{B}_{\alpha}^{\beta} \mathcal{D} B_{\alpha}^{\beta} \delta\left(B_{\alpha}^{\beta}-\frac{2 g_{2}}{3}\left(\bar{q}^{c} \Gamma_{\alpha} q\right) P^{\beta} q\right) \delta\left(\bar{B}_{\alpha}^{\beta}-\frac{2 g_{2}}{3} \bar{q} P^{\beta}\left(\bar{q} \Gamma_{\alpha} q^{c}\right)\right) \\
= & \int \mathcal{D} \bar{B}_{\alpha}^{\beta} \mathcal{D} B_{\alpha}^{\beta} \mathcal{D} \bar{\Psi}_{\beta}^{\alpha} \mathcal{D} \Psi_{\beta}^{\alpha} \\
& \quad \exp \left(i \int\left[\left\{B_{\alpha}^{\beta}-\frac{2 g_{2}}{3}\left(\bar{q}^{c} \Gamma_{\alpha} q\right) P^{\beta} q\right\} \bar{\Psi}_{\beta}^{\alpha}+\Psi_{\beta}^{\alpha}\left\{\bar{B}_{\alpha}^{\beta}-\frac{2 g_{2}}{3} \bar{q} P^{\beta}\left(\bar{q} \Gamma_{\alpha} q^{c}\right)\right\}\right]\right) .
\end{aligned}
$$

Note, that we are working in Euclidean space where we have used the Wick rotation $t \rightarrow-i \tau$. For notational simplification we use the abbreviation $\int=\int d^{4} x=\int d^{3} \boldsymbol{r} d \tau$ in the exponents. The operator $P^{\beta}$ projects the three quarks onto the quantum numbers of the considered physical baryon states. In particular, the color of the third quark has to be chosen to build a colorless baryon wavefunction.

As intermediate building blocks for the baryon field we introduce also diquark fields

$$
\begin{aligned}
\mathbb{1} & =\int \mathcal{D} \kappa_{\alpha} \mathcal{D} \kappa_{\alpha}^{*} \delta\left(\kappa_{\alpha}^{*}-\bar{q} \Gamma_{\alpha} q^{c}\right) \delta\left(\kappa_{\alpha}-\bar{q}^{c} \Gamma_{\alpha} q\right) \\
& =\int \mathcal{D} \kappa_{\alpha} \mathcal{D} \kappa_{\alpha}^{*} \mathcal{D} \Delta^{\alpha} \mathcal{D} \Delta^{\alpha *} \exp \left(\frac{i}{2} \int\left[\left(\kappa_{\alpha}^{*}-\bar{q} \Gamma_{\alpha} q^{c}\right) \Delta^{\alpha}+\Delta^{\alpha *}\left(\kappa_{\alpha}-\bar{q}^{c} \Gamma_{\alpha} q\right)\right]\right) .
\end{aligned}
$$

Replacing all terms of fourth order in the quark fields with help of the constraints (2.6) and (2.8), and integrating out the auxiliary fields $\chi_{\alpha}, \kappa_{\alpha}$ and $\kappa_{\alpha}^{*}$, one obtains the following form for the generating functional

$$
\begin{aligned}
Z \sim & \int \mathcal{D} q \mathcal{D} \bar{q} \mathcal{D} \varphi \mathcal{D} \Delta \mathcal{D} \tilde{\Delta} \mathcal{D} \bar{B} \mathcal{D} B \mathcal{D} \bar{\Psi}^{\alpha} \mathcal{D} \Psi^{\alpha} \\
& \exp \left(\int\left[\bar{q}\left\{i \gamma^{\mu} \partial_{\mu}-m^{0}-\varphi+\frac{4 g_{2}}{3} \bar{\Psi}^{\alpha} \Psi^{\alpha}\right\} q+\bar{q} \Delta_{\alpha}^{*} \Psi^{\alpha}+\bar{\Psi}^{\alpha} \Delta_{\alpha} q\right]\right) \\
& * \exp \left(-\int\left[\frac{1}{2} \bar{q} \Delta q^{c}+\frac{1}{2} \bar{q}^{c} \tilde{\Delta} q+\frac{1}{8 g_{1}} \operatorname{tr} \varphi^{2}+\frac{3}{8 g_{2}} \operatorname{tr} \tilde{\Delta} \Delta-i\left\{\bar{\Psi}_{\beta}^{\alpha} B_{\alpha}^{\beta}+\bar{B}_{\alpha}^{\beta} \Psi_{\beta}^{\alpha}\right\}\right]\right)
\end{aligned}
$$

\footnotetext{
${ }^{1}$ Similar hadronization approaches have been considered in [31,32]
} 
where we have introduced the compact matrix notation for the meson and diquark fields $\varphi=\varphi_{\alpha} \Lambda^{\alpha}, \Delta=\Gamma_{\alpha} \Delta^{\alpha}, \tilde{\Delta}=\Gamma_{\alpha} \Delta^{\alpha *}$ as well as for the baryon sources $\Psi^{\alpha}=\Psi_{\beta}^{\alpha} P^{\beta}$ and $\bar{\Psi}^{\alpha}=P^{\beta} \bar{\Psi}_{\beta}^{\alpha}$. The symbol tr corresponds to the trace over color, flavor and Dirac spinor degrees of freedom.

To eliminate the quark degrees of freedom we are working within the Nambu-Gorkov formalism developed originally in the theory of superconductivity [33 35]. For that purpose we introduce combined Grassmann fieldst

$$
\mathbf{q}=\left(\begin{array}{c}
q \\
\bar{q}^{T}
\end{array}\right) \quad, \quad \overline{\mathbf{q}}=\left(\bar{q}, q^{T}\right)
$$

for the quark fields and

$$
\Psi^{\alpha}=\left(\begin{array}{c}
\Psi^{\alpha} \\
\bar{\Psi}^{\alpha T}
\end{array}\right) \quad, \quad \bar{\Psi}^{\alpha}=\left(\bar{\Psi}^{\alpha}, \Psi^{\alpha T}\right)
$$

for the baryon sources. Now, we integrate out the quark fields $\mathbf{q}$ and $\overline{\mathbf{q}}$ with help of the Nambu-Gorkov formula

$$
\begin{aligned}
& \int \mathcal{D} q \mathcal{D} \bar{q} \exp \left(\bar{q} \mathcal{G}^{-1} q-\frac{1}{2} \bar{q} \Delta q^{c}-\frac{1}{2} \bar{q}^{c} \tilde{\Delta} q+\left[\bar{q} \Delta^{* \alpha} \Psi_{\alpha}+\bar{\Psi}_{\alpha} \Delta^{\alpha} q\right]\right) \\
= & \int \mathcal{D} \mathbf{q} \mathcal{D} \overline{\mathbf{q}} \exp \left(\frac{1}{2}\left[\overline{\mathbf{q}} \mathbf{G}^{-1} \mathbf{q}+\overline{\mathbf{q}} \boldsymbol{\xi}+\overline{\boldsymbol{\xi}} \mathbf{q}\right]\right)=\left(\operatorname{DetG}^{-1}\right)^{\frac{1}{2}} \exp \left(\frac{1}{2} \overline{\boldsymbol{\xi}} \mathbf{G} \boldsymbol{\xi}\right),
\end{aligned}
$$

where we have defined

$$
\boldsymbol{\xi}=\left(\begin{array}{c}
\Delta^{* \alpha} \Psi_{\alpha} \\
-\bar{\Psi}_{\alpha}^{T} \Delta^{\alpha}
\end{array}\right) \quad, \quad \overline{\boldsymbol{\xi}}=\left(\bar{\Psi}_{\alpha} \Delta^{\alpha},-\Delta^{* \alpha} \Psi_{\alpha}^{T}\right),
$$

and the inverse quark Greens function

$$
\mathbf{G}^{-1}=\left(\begin{array}{cc}
\mathcal{G}^{-1} & -\Delta C \\
-C \Delta^{*} & -V \tilde{\mathcal{G}}^{-1} V^{\dagger}
\end{array}\right), \quad \tilde{\mathcal{G}}=V^{\dagger} \mathcal{G}^{T} V .
$$

The off-diagonal elements of $\mathbf{G}$ are the so-called anomalous Greens functions which are related to the amplitude for adding or subtracting a pair of quarks to the system. The transformation operator $V=J G$, which we have introduced for technical reasons, is a combination of the self-adjoint unitary transformation $J=i \beta \gamma_{5}$ and the G-parity operator $G=e^{i \pi \tau_{2} / 2} C$.

\footnotetext{
${ }^{2}$ Quantities in the Nambu-Gorkov formalism are denoted by boldface letters.
} 
The normal quark Greens function is represented by

$$
\mathcal{G}^{-1}=i \gamma^{\mu} \partial_{\mu}-\varphi+\frac{4 g_{2}}{3} \bar{\Psi}^{\alpha} \Psi^{\alpha}=\mathcal{G}_{0}^{-1}+\frac{4 g_{2}}{3} \bar{\Psi}^{\alpha} \Psi^{\alpha}
$$

From eq. (2.13) we observe that the baryon sources $\Psi^{\alpha}$ are contracted with the diquark fields $\Delta_{\alpha}$. Hence these sources only couple to a single quark. Since in the ladder approximation (described below) a three quark bound state cannot be affected by such a coupling it is sufficient to restrict the quark Greens function to $\mathcal{G}_{0}^{-1}$.

Finally, the generating functional is given by

$$
\begin{aligned}
Z\left[B_{\alpha}^{\beta}, \bar{B}_{\alpha}^{\beta}\right] \sim \int \mathcal{D} \varphi \mathcal{D} \Delta \mathcal{D} \tilde{\Delta} \mathcal{D} \bar{\Psi}^{\alpha} \mathcal{D} \Psi^{\alpha} \\
\quad \exp \left(\mathcal{A}\left[\varphi, \Delta, \tilde{\Delta}, \bar{\Psi}^{\alpha}, \Psi^{\alpha}\right]+i\left(\bar{\Psi}_{\beta}^{\alpha} B_{\alpha}^{\beta}+\bar{B}_{\alpha}^{\beta} \Psi_{\beta}^{\alpha}\right)\right)
\end{aligned}
$$

with the effective action

$$
\begin{aligned}
& \mathcal{A}\left[\varphi, \Delta, \tilde{\Delta}, \bar{\Psi}^{\alpha}, \Psi^{\alpha}\right]= \\
& \mathcal{A}_{q}\left[\varphi, \Delta, \tilde{\Delta}, \bar{\Psi}^{\alpha}, \Psi^{\alpha}\right]+\mathcal{A}_{v a l}\left[\varphi, \Delta, \tilde{\Delta}, \bar{\Psi}^{\alpha}, \Psi^{\alpha}\right]+\mathcal{A}_{m}[\varphi]+\mathcal{A}_{d}[\Delta, \tilde{\Delta}] .
\end{aligned}
$$

The first part, the so-called quark determinant,

$$
\mathcal{A}_{q}=\frac{1}{2} \operatorname{Tr} \log \mathbf{G}^{-1}
$$

carries the full information of the underlying quark spectrum via the functional trace Tr. The second part

$$
\mathcal{A}_{v a l}=\frac{1}{2} \overline{\boldsymbol{\Psi}}^{\alpha}\left(\mathbf{G}_{B}^{v a l}\right)_{\alpha \beta} \mathbf{\Psi}^{\beta}
$$

contains the valence quark part of the baryon propagator $\left(\mathbf{G}_{B}^{v a l}\right)_{\alpha \beta}$ originating from the inversion of the quark propagator $\mathbf{G}_{0}$ ( $c f$. appendix B for the derivation of $\left(\mathbf{G}_{B}^{v a l}\right)_{\alpha \beta}$.).

Both residual terms

$$
\begin{aligned}
\mathcal{A}_{m} & =-\frac{1}{8 g_{1}} \int d^{4} x\left[\operatorname{tr} \varphi^{2}\right] \\
\mathcal{A}_{d} & =-\frac{3}{8 g_{2}} \int d^{4} x[\operatorname{tr} \tilde{\Delta} \Delta]
\end{aligned}
$$

are pure mass terms for the meson and diquark fields, respectively"

\footnotetext{
${ }^{3}$ Note the additional factor $N_{C}=3$ in the diquark mass term $\mathcal{A}_{d}$ as compared to the mass term $\mathcal{A}_{m}$ of the mesons.
} 


\section{TWO AND THREE QUARK CORRELATIONS IN A SOLITONIC BACKGROUND}

\section{A. The static soliton background}

In this section we are interested in the behavior of quark-quark and diquark-quark bound states in the background field of a soliton configuration. In the first step we neglect the explicit baryon sources. With this restriction it is possible to expand the effective action (2.17)

$$
\mathcal{A}\left[\varphi, \Delta, \tilde{\Delta}, \bar{\Psi}^{\alpha}=0, \Psi^{\alpha}=0\right]=\mathcal{A}^{(0)}[\varphi]+\mathcal{A}^{(1)}[\varphi, \Delta, \tilde{\Delta}]+\mathcal{A}^{(2)}[\varphi, \Delta, \tilde{\Delta}]+\ldots
$$

up to second order in the diquark fields where (see also eqs. (2.19) and (2.20))

$$
\begin{aligned}
\mathcal{A}^{(0)}[\varphi] & =\mathcal{A}_{q}^{(0)}+\mathcal{A}_{\text {val }}+\mathcal{A}_{m}, \\
\mathcal{A}^{(1)}[\varphi, \Delta, \tilde{\Delta}] & =\mathcal{A}_{q}^{(1)}, \\
\mathcal{A}^{(2)}[\varphi, \Delta, \tilde{\Delta}] & =\mathcal{A}_{q}^{(2)}+\mathcal{A}_{d} .
\end{aligned}
$$

Within Schwinger's proper time regularization description [36] the parts stemming from the quark determinant (2.18) can be written as

$$
\begin{aligned}
& \mathcal{A}_{q}^{(0)}=-\frac{1}{4} \operatorname{Tr} \int_{1 / \Lambda^{2}}^{\infty} \frac{d s}{s} e^{-s \mathbf{A}_{0}}, \\
& \mathcal{A}_{q}^{(1)}=\frac{1}{4} \operatorname{Tr} \int_{1 / \Lambda^{2}}^{\infty} d s \mathbf{A}_{1} e^{-s \mathbf{A}_{0}}, \\
& \mathcal{A}_{q}^{(2)}=\frac{1}{4} \operatorname{Tr} \int_{1 / \Lambda^{2}}^{\infty} d s \mathbf{A}_{2} e^{-s \mathbf{A}_{0}}-\frac{1}{4} \operatorname{Tr} \int_{1 / \Lambda^{2}}^{\infty} d s s \int_{0}^{1} d \alpha \alpha \mathbf{A}_{1} e^{-s \alpha \mathbf{A}_{0}} \mathbf{A}_{1} e^{-s(1-\alpha) \mathbf{A}_{0}} .
\end{aligned}
$$

The Nambu-Gorkov matrices $\mathbf{A}_{i}$ originating from the expansion of the operator $\left(\mathbf{G}^{-1}\right)^{\dagger} \mathbf{G}^{-1}=\mathbf{A}_{0}+\mathbf{A}_{1}+\mathbf{A}_{2}$ have the explicit form

$$
\begin{aligned}
& \mathbf{A}_{0}=\left(\begin{array}{cc}
\left(\mathcal{G}^{-1}\right)^{\dagger} \mathcal{G}^{-1} & 0 \\
0 & V^{-1}\left(\tilde{\mathcal{G}}^{-1}\right)^{\dagger} \tilde{\mathcal{G}}^{-1} V
\end{array}\right), \\
& \mathbf{A}_{1}=\left(\begin{array}{cc}
0 & -\left(\mathcal{G}^{-1}\right)^{\dagger} \Delta C-\tilde{\Delta}^{\dagger} C V^{-1} \tilde{\mathcal{G}}^{-1} V \\
C \Delta^{\dagger} \mathcal{G}^{-1}+V^{-1}\left(\tilde{\mathcal{G}}^{-1}\right)^{\dagger} V C \tilde{\Delta} & 0
\end{array}\right), \\
& \mathbf{A}_{2}=\left(\begin{array}{cc}
\tilde{\Delta}^{\dagger} \tilde{\Delta} & 0 \\
0 & -C \Delta^{\dagger} \Delta C
\end{array}\right) .
\end{aligned}
$$

By expanding $\mathcal{A}_{q}^{(0)}$ to quadratic order in $\varphi$ we can relate [21,37] the parameters $\left(g_{1}\right.$, $m_{0}=m_{0}^{u}=m_{0}^{d}$ and $\Lambda$ ) of the model to the pion mass $m_{\pi}=135 \mathrm{MeV}$ and the pion decay 
constant $f_{\pi}=93 \mathrm{MeV}$, leaving undetermined just one parameter which we choose to be the constituent quark mass $m$. This mass is the vacuum expectation value of the scalar field and reflects the spontaneous breaking of chiral symmetry. In principle the diquark coupling constant $g_{2}$ is fixed by the Fierz transformation, however, we will relax the restriction $g_{2} / g_{1}=1$ thus effectively treating $g_{2} / g_{1}$ as a parameter to study the influence of the diquarks.

Note, that for each term in eq. (3.5) the Nambu-Gorkov trace can be worked out analytically. In addition, due to the diagonality of $\mathbf{A}_{0}$ and the vanishing trace of $\mathbf{A}_{1}$ it is obvious that $\Delta=\tilde{\Delta}=0$ is always a solution of the equation of motion though it is not necessarily the solution of least action. For the remaining functional trace we use the color degenerated eigenstates of the inverse propagator for quarks in the background of a static meson field $\varphi$

$$
\mathcal{G}^{-1}|n, \nu\rangle=(i \not \partial-\varphi)|n, \nu\rangle=\beta\left(-\partial_{\tau}-h_{\Theta}\right)|n, \nu\rangle=\beta\left(i \omega_{n}-\epsilon_{\nu}\right)|n, \nu\rangle
$$

and perform an additional color trace $\operatorname{tr}_{C}$. In analogy, the charge conjugated quark propagator $\tilde{\mathcal{G}}_{0}^{-1}$ can be worked out similarly:

$$
\tilde{\mathcal{G}}_{0}^{-1}|n, \nu\rangle=V^{\dagger}(i \not \partial-\varphi)^{T} V|n, \nu\rangle=\beta\left(\partial_{\tau}-h_{\Theta}\right)|n, \nu\rangle=\beta\left(-i \omega_{n}-\epsilon_{\nu}\right)|n, \nu\rangle .
$$

For the meson fields we make use of the polar decomposition

$$
\varphi=\Phi U^{\gamma_{5}}=m U^{\gamma_{5}}
$$

where we have fixed the chiral radius $\Phi$ to its vacuum expectation value $m$. Additionally, we adopt the well-known hedgehog ansatz for the chiral field

$$
U=\exp (i \boldsymbol{\tau} \cdot \hat{\boldsymbol{r}} \Theta(r))
$$

Due to the static field configuration it is possible to extract from the zero order term $\mathcal{A}^{(0)}=-T E_{\text {sol }}[\Theta](3.2)$ an energy functional

$$
\begin{aligned}
E_{\text {sol }}[\Theta] & =E_{\text {sea }}+E_{\text {val }} \\
E_{\text {sea }} & =\frac{3}{2} \int_{1 / \Lambda^{2}}^{\infty} d s \frac{1}{\sqrt{4 \pi s^{3}}} \sum_{\mu} e^{-s \epsilon_{\mu}^{2}}+m_{\pi}^{2} f_{\pi}^{2} \int d^{3} r(1-\cos \Theta(r)) \\
E_{\text {val }} & =\frac{3}{2} \epsilon_{\text {val }}\left(1+\operatorname{sgn}\left(\epsilon_{\text {val }}\right)\right) .
\end{aligned}
$$

Neglecting quark-quark correlations, the valence quark contribution $E_{\text {val }}$ is composed of the color degenerated single particle energies of the valence quark level. This state is the only one which is bound by the soliton, i.e. $-m<\epsilon_{v a l}<m$. The soliton configuration is characterized by the chiral angle $\Theta_{s c}(r)$ which is self-consistently determined by extremizing the energy functional $E[\Theta]$ [38 40 . 


\section{B. Bethe-Salpeter equation for diquarks}

In the following we derive the equation of motion for the diquark fields in the solitonic meson background field. The solutions of this equation define the eigenmodes $b_{\alpha}$ and $b_{\alpha}^{*}$ of the diquark fields

$$
\begin{aligned}
& \Delta=b_{\alpha}\left(\Delta(\boldsymbol{r}, t) \Gamma C V^{-1}\right)^{\alpha}=b_{\alpha} \Gamma_{d i q}^{\alpha}(\boldsymbol{r}, t), \\
& \tilde{\Delta}=(V C \Gamma \Delta(\boldsymbol{r}, t))^{\alpha} b_{\alpha}^{*}=b_{\alpha}^{*} \tilde{\Gamma}_{d i q}^{\alpha}(\boldsymbol{r}, t)
\end{aligned}
$$

which we have redefined including the matrices $V$ and $C$ into the diquark vertices. For the present qualitative discussion it is sufficient to consider only an S-wave scalar diquark field. In respect of the Pauli principle the only possible ansatz for the vertex functions $\Gamma_{d i q}^{\alpha}(\boldsymbol{r}, t)$ is

$$
\Gamma_{d i q}^{\alpha}(\boldsymbol{r}, t)=\Delta(r, t) \Gamma^{\alpha} \quad, \quad \tilde{\Gamma}_{d i q}^{\alpha}(\boldsymbol{r}, t)=\Delta^{*}(r, t) \Gamma^{\alpha} \quad, \quad \Gamma^{\alpha}=-\frac{\lambda_{C}^{a}}{\sqrt{2}} \frac{\tau_{2}}{2} i \gamma_{5}
$$

with $\lambda_{C}^{a}(a=2,5,7)$ being the antisymmetric Gell-Mann matrices of the color group.

Using the Fourier-transform in the time coordinate

$$
\Delta(r, \tau)=\int \frac{d \omega}{2 \pi} \Delta(r, i \omega) e^{-i \omega \tau}
$$

and the spatial representation $\Psi_{\nu}(\boldsymbol{r})=\langle\boldsymbol{r} \mid \nu\rangle$ for the quark eigenstates the residual trace can be worked out. The second order term of the effective action, $\mathcal{A}_{q}^{(2)}$, together with $\mathcal{A}_{d}$

determines the Bethe-Salpeter equation as the equation of motion for the diquark fields $\delta \mathcal{A}^{(2)} / \delta \Delta^{*}(r,-i \omega)=0$ :

$$
\left.r^{2}\left[\int d r^{\prime} r^{\prime 2} D_{\text {diq }}^{-1}\left(r, r^{\prime} ; i \omega\right) \Delta_{\alpha}\left(r^{\prime}, i \omega\right)\right]\right|_{\omega^{2}=-\omega_{d i q}^{2}}=0
$$

The inverse diquark propagator

$$
D_{\mathrm{diq}}^{-1}\left(r, r^{\prime} ; i \omega\right)=K\left(r, r^{\prime} ; i \omega\right)-\frac{\pi m_{\pi}^{2} f_{\pi}^{2}}{2 m_{0} m} \delta\left(r-r^{\prime}\right)
$$

is expressed in terms of a local mass term and a bilocal kernel

$$
K\left(r^{\prime}, r ; i \omega\right)=\sum_{\nu \mu} \int d \Omega d \Omega^{\prime} R\left(i \omega ; \epsilon_{\nu}, \epsilon_{\mu}\right)\left[\Psi_{\nu}^{\dagger}\left(\boldsymbol{r}^{\prime}\right) \Psi_{\mu}\left(\boldsymbol{r}^{\prime}\right) \Psi_{\mu}^{\dagger}(\boldsymbol{r}) \Psi_{\nu}(\boldsymbol{r})\right]
$$

where

$$
\begin{aligned}
R\left(i \omega ; \epsilon_{\nu}, \epsilon_{\mu}\right) & =\frac{1}{16 \sqrt{\pi}}\left[\frac{1}{2\left|\epsilon_{\nu}\right|} \Gamma\left(\frac{1}{2}, \frac{\epsilon_{\nu}^{2}}{\Lambda^{2}}\right)+\frac{1}{2\left|\epsilon_{\mu}\right|} \Gamma\left(\frac{1}{2}, \frac{\epsilon_{\mu}^{2}}{\Lambda^{2}}\right)\right. \\
& \left.-\left(\omega^{2}+\left(\epsilon_{\nu}-\epsilon_{\mu}\right)^{2}\right) \int_{0}^{1} d \alpha \alpha \frac{\Gamma\left(\frac{3}{2},\left[\alpha \epsilon_{\mu}^{2}+(1-\alpha) \epsilon_{\nu}^{2}+\alpha(1-\alpha) \omega^{2} / \Lambda^{2}\right]\right)}{\left[\alpha \epsilon_{\mu}^{2}+(1-\alpha) \epsilon_{\nu}^{2}+\alpha(1-\alpha) \omega^{2}\right]^{3 / 2}}\right]
\end{aligned}
$$


in the proper-time regularized version of the quark loop.

For the ongoing discussion a normalization of the diquark field $\Delta_{\alpha}(r, i \omega)$ is necessary. In principle such a normalization would be obtained in the framework of second quantization. Equivalently, we demand that the total baryon charge, to which the diquarks contribute, equals unity. For that purpose we first calculate the expectation value of the baryon number operator $\hat{B}=\mathbb{1}_{c} / N_{c}=\mathbb{1}_{c} / 3$ (see appendix $\mathrm{C}$ for details.). In analogy to the effective action (2.17) the expression (C5) is expanded in terms of diquark fields. The leading order yields the value $1 / 3$, which is nothing but the contribution of a single quark. Therefore, we require for the second order term the normalization condition

$$
B_{d i q} \stackrel{!}{=} \frac{2}{3}
$$

with $B_{d i q}$ defined in (C10). As the solutions of the Bethe-Salpeter equation appear always in pairs $\pm i \omega_{d i q}$, the normalization condition (3.21) allows us to distinguish between diquark and antidiquark solutions.

\section{Faddeev equation for a quark-diquark bound state}

Up to this point, we have dealt with the generating functional (2.16) for meson, diquark and baryon fields. Below we derive an effective meson-baryon action generalizing the approach of 29]. For this purpose we perform the diquark integration. Since the diquark fields appear to all orders we introduce external diquark sources $j, \tilde{j}$ and rewrite $Z \sim$ $\int \mathcal{D} \varphi \mathcal{D} \bar{\Psi}^{\alpha} \mathcal{D} \Psi^{\alpha} Z_{d i q}[j, \tilde{j}]$ (2.16) in the form

$$
\begin{aligned}
Z_{\text {diq }}[j, \tilde{j}] & \sim \int \mathcal{D} \Delta \mathcal{D} \tilde{\Delta} \exp \left(\mathcal{A}^{(2)}[\Delta, \tilde{\Delta}]+\mathcal{A}_{\text {int }}[\Delta, \tilde{\Delta}]+i[\tilde{\Delta} j+\tilde{j} \Delta]\right) \\
& \sim \exp \left(\mathcal{A}_{i n t}\left[\frac{\delta}{i \delta \tilde{j}}, \frac{\delta}{i \delta j}\right]\right) \int \mathcal{D} \Delta \mathcal{D} \tilde{\Delta} \exp \left(\tilde{\Delta} D_{d i q}^{-1} \Delta+i(\tilde{\Delta} j+\tilde{j} \Delta)\right) .
\end{aligned}
$$

The integration over the diquark fields results in

$$
Z_{d i q}[j, \tilde{j}] \sim \exp \left(\mathcal{A}_{v a l}\left[\frac{\delta}{i \delta \tilde{j}}, \frac{\delta}{i \delta j}\right]\right) \exp \left(\tilde{j} D_{d i q} j\right)
$$

Note, that the interaction part $\mathcal{A}_{\text {int }}=\mathcal{A}_{\text {val }}$ is completely determined by the valence quark part of the baryon propagator. Using the geometric series

$$
\begin{aligned}
\left(1+\mathcal{G}_{0} \Delta \tilde{\mathcal{G}_{0}} \tilde{\Delta}\right)^{-1} & =\left(1+\mathcal{G}_{0} b_{\gamma} \Gamma_{d i q}^{\gamma} \tilde{\mathcal{G}}_{0} \tilde{\Gamma}_{d i q}^{\delta} b_{\delta}^{*}\right)^{-1} \\
& =\sum_{a=0}^{\infty}\left(\mathcal{G}_{0} b_{\gamma} \Gamma_{d i q}^{\gamma} \tilde{\mathcal{G}}_{0} \tilde{\Gamma}_{d i q}^{\delta} b_{\delta}^{*}\right)^{a}
\end{aligned}
$$

to reexpress $G_{11}$ in eq. (B4) we obtain the baryon propagator 

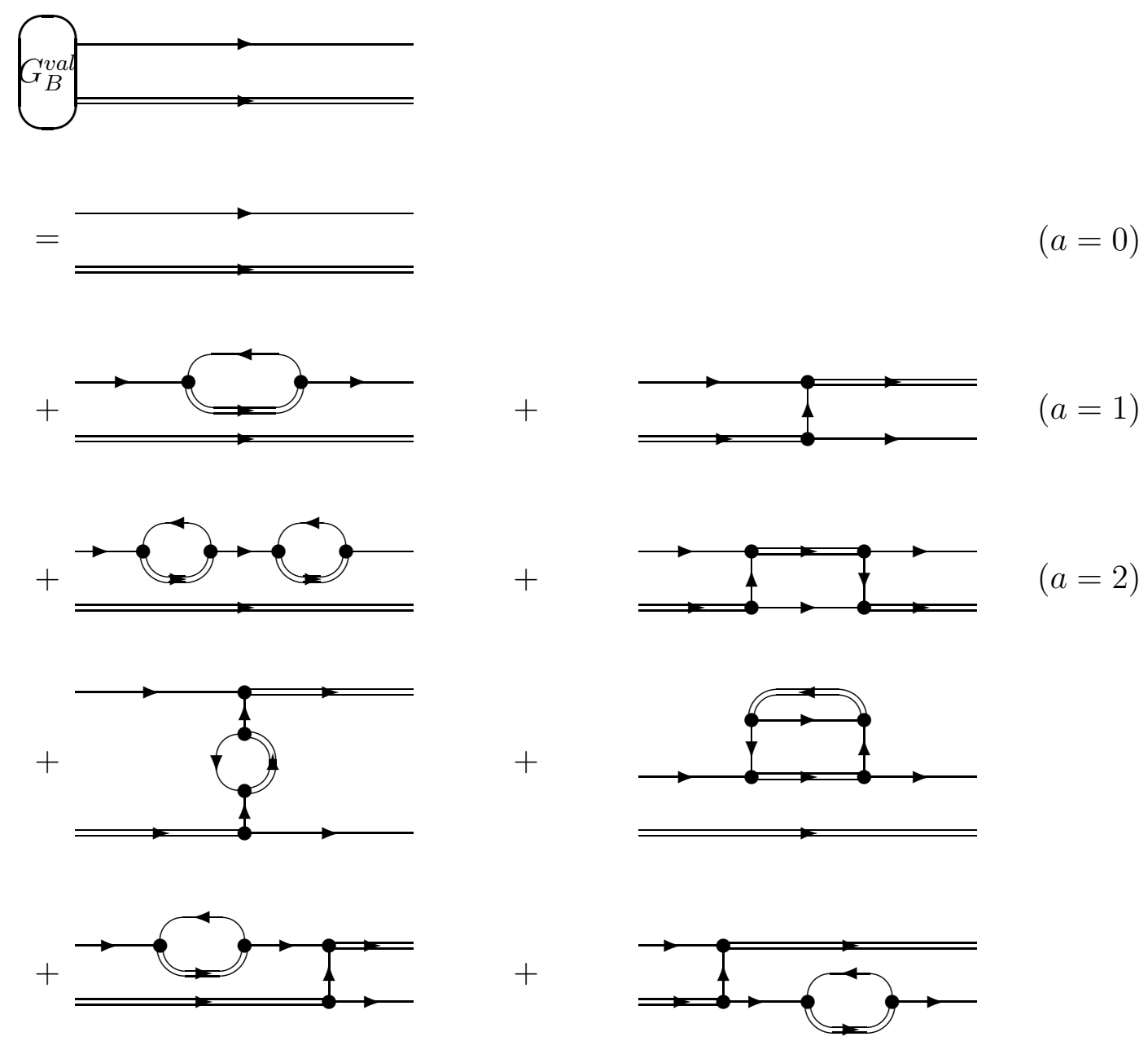

$+\ldots$

$$
(a \geq 3)
$$

FIG. 1. Feynman diagrams of the series (3.25) for the baryon propagator $G_{B}^{v a l}$

$$
\begin{aligned}
\left(G_{B}^{v a l}\right)_{\alpha \beta}=\mathcal{G}_{0} D_{\alpha \beta} & +\mathcal{G}_{0} D_{\gamma \delta} \Gamma_{d i q}^{\gamma} \tilde{\mathcal{G}}_{0} \tilde{\Gamma}_{d i q}^{\delta} D_{\alpha \beta} \mathcal{G}_{0} \\
& +\mathcal{G}_{0} D_{\alpha \delta} \Gamma_{d i q}^{\gamma} \tilde{\mathcal{G}}_{0} \tilde{\Gamma}_{d i q}^{\delta} D_{\gamma \beta} \mathcal{G}_{0}+\ldots
\end{aligned}
$$

as an infinite series in quark $\mathcal{G}_{0}$ and diquark propagators $D_{\alpha \beta}$. This series is drawn as a sum of Feynman diagrams in Fig. 1. For the calculation of bound states it is only necessary to consider the quark exchange graphs in the series (3.25). Neglecting the self-interaction graphs is justified because we have an effective interaction and therefore all self-interaction graphs can be absorbed in the effective coupling constants. This simplification is nothing else than the well-known ladder approximation which has the advantage that the baryon propagator can summed up completely: 


$$
\begin{aligned}
\left(G_{B}^{v a l}\right)_{\alpha \beta}= & \mathcal{G}_{0} D_{\alpha \beta}+\mathcal{G}_{0} D_{\alpha \delta} \Gamma_{d i q}^{\gamma} \tilde{\mathcal{G}}_{0} \tilde{\Gamma}_{d i q}^{\delta} D_{\gamma \beta} \mathcal{G}_{0} \\
& +\mathcal{G}_{0} D_{\alpha \delta} \Gamma_{d i q}^{\gamma} \tilde{\mathcal{G}}_{0} \tilde{\Gamma}_{d i q}^{\delta} D_{\gamma \eta} \Gamma_{d i q}^{\epsilon} \tilde{\mathcal{G}}_{0} \tilde{\Gamma}_{d i q}^{\eta} D_{\epsilon \beta} \mathcal{G}_{0}+\ldots \\
= & \left(G_{B}^{0}\right)_{\alpha \beta}+\left(G_{B}^{0}\right)_{\alpha \delta} H^{\gamma \delta}\left(G_{B}^{0}\right)_{\gamma \beta}+\left(G_{B}^{0}\right)_{\alpha \delta} H^{\gamma \delta}\left(G_{B}^{0}\right)_{\gamma \eta} H^{\epsilon \eta}\left(G_{B}^{0}\right)_{\epsilon \beta}+\ldots \\
= & \left(1-\left(G_{B}^{0}\right)_{\alpha \delta} H^{\gamma \delta}\right)^{-1}\left(G_{B}^{0}\right)_{\gamma \beta} .
\end{aligned}
$$

Here we have introduced the free quark-diquark propagator

$$
\left(G_{B}^{0}\right)_{\alpha \beta}=\mathcal{G}_{0} D_{\alpha \beta}
$$

and the quark exchange operator

$$
H^{\gamma \delta}=\Gamma_{d i q}^{\gamma} \tilde{\mathcal{G}}_{0} \tilde{\Gamma}_{d i q}^{\delta}
$$

We are now able to integrate over the baryon source fields $\Psi^{\alpha}, \bar{\Psi}^{\alpha}$. This leads to an effective baryon action

$$
\mathcal{A}_{\text {bary }}^{(2)}=\bar{B}^{\alpha}\left(G_{B}^{v a l}\right)_{\alpha \beta}^{-1} B^{\beta}
$$

for the baryon fields $\bar{B}^{\alpha}=P^{\nu} \bar{B}_{\nu}^{\alpha}$ und $B^{\alpha}=B_{\nu}^{\alpha} P^{\nu}$. The inversion of the baryon propagator

$$
\left(G_{B}^{v a l}\right)_{\alpha \beta}^{-1}=\left(G_{B}^{0}\right)_{\alpha \beta}^{-1}-H_{\alpha \beta}
$$

follows directly from eq. (3.26). Using

$$
\frac{\delta \mathcal{A}_{b a r y}^{(2)}}{\delta \bar{B}^{\alpha}}=0
$$

one finally arrives at the Faddeev equation in operator form:

$$
\left(G_{B}^{v a l}\right)_{\alpha \beta}^{-1} B^{\beta}=0
$$

In the next step we construct the kernel of the matrix $\left(G_{B}^{v a l}\right)_{\alpha \beta}^{-1}$. For this purpose we expand the baryon field

$$
B(x, y)=\int d \omega d E \sum_{\alpha} \sum_{\nu}^{\left(\epsilon_{\nu}>0\right)} a_{\alpha \nu}(E, \omega) B_{\alpha \nu}^{0}\left(\vec{x}, x_{4} ; \vec{y}, y_{4}\right)
$$

in eigenfunctions

$$
B_{\alpha \nu}^{0}\left(\vec{x}, x_{4} ; \vec{y}, y_{4}\right)=\frac{1}{(2 \pi)^{2}} \Delta_{\alpha}(\vec{x}) e^{-i \omega x_{4}} \Psi_{\nu}(\vec{y}) e^{-i E y_{4}}
$$

of the free quark-diquark propagator

$$
\left(G_{B}^{0}\right)_{\alpha \beta}^{-1} B_{\beta \nu}^{0}\left(\vec{x}, x_{4} ; \vec{y}, y_{4}\right)=-\beta\left(i E-\epsilon_{\nu}\right)\left(\omega^{2}+\omega_{\beta}^{2}\right) B_{\beta \nu}^{0}\left(\vec{x}, x_{4} ; \vec{y}, y_{4}\right) .
$$


The projection operator $P^{\beta}$ (2.7) on the third quark projects out the positive energy eigenstates $\left(\epsilon_{\nu}>0\right)$. Transforming the energy variables $E, \omega$ to the total energy $\Omega=E+\omega$ and the relative energy $\tilde{\Omega}=\frac{1}{2}(\omega-E)$ we obtain the Faddeev equation in terms of the coefficients $a_{\alpha \nu}(i \Omega, i \tilde{\Omega})$ :

$$
\begin{aligned}
{\left[\frac{1}{2} i \Omega-i \tilde{\Omega}-\epsilon_{\nu}\right][} & \left.\left(\frac{1}{2} \Omega+\tilde{\Omega}\right)^{2}+\omega_{\alpha}^{2}\right] a_{\alpha \nu}(i \Omega, i \tilde{\Omega}) \\
& +\frac{1}{4} \int d \tilde{\Omega}^{\prime} \sum_{\beta, \mu, \kappa} \frac{\left\langle\nu\left|\Gamma_{d i q}^{\beta}\right| \kappa\right\rangle\left\langle\kappa\left|\Gamma_{d i q}^{\alpha}\right| \mu\right\rangle}{i\left(\tilde{\Omega}+\tilde{\Omega}^{\prime}\right)+\epsilon_{\kappa}} a_{\beta \mu}\left(i \Omega, i \tilde{\Omega}^{\prime}\right)=0 .
\end{aligned}
$$

The summation runs over the one-particle quark eigenstates $|\mu\rangle$ and $|\kappa\rangle$ of $h_{\Theta}$. To reduce the numerical effort we restrict the diquark states to the energetically lowest state $\Gamma_{d i q}^{\alpha}=$ $\Gamma_{d i q}$ t. In fact, this is the only bound state; all other states lie above the two quark threshold and, if at all, influence the quark-diquark bound state only weakly.

It is important to equip the absolute and relative energies, $\Omega$ and $\tilde{\Omega}$, respectively with a physical meaning. These quantities do not yet have apparent interpretations since due to the presence of the static and localized soliton Lorentz covariance is lost. Hence we are still lacking an energy scale. In order to define a physically relevant energy scale we consider the limit of vanishing diquark-quark coupling $\left(\Gamma_{d i q} \rightarrow 0\right)$. In this limit the different quark states decouple and the Faddeev equation reduces to a purely algebraic equation. For the valence quark part we have the three solutions $i \Omega=2\left(i \tilde{\Omega}+\epsilon_{\text {val }}\right)$ and $i \Omega=4\left(-i \tilde{\Omega} \pm \omega_{\text {diq }}\right)$. When the energy transfer vanishes, i.e. $\tilde{\Omega}=0$, we should obtain the physically reasonable solution $i \Omega=\omega_{d i q}+\epsilon_{v a l}$ for the energy of a free baryon. This result can only be realized if we enforce the variable substitution $\tilde{\Omega} \rightarrow \tilde{\Omega}+\frac{i}{2}\left(\epsilon_{v a l}-\omega_{d i q}\right)$. Thus, two of the three solutions join the physical solution. The third one yielding a negative solution is rejected because we have projected to positive energy states. Since the limit of vanishing diquarkquark coupling should continuously emerge from the complete solution of the Faddeev equation it is necessary to perform the variable substitution $\tilde{\Omega} \tilde{\Omega} \rightarrow \tilde{\Omega}+\frac{i}{2}\left(\epsilon_{\text {val }}-\omega_{\text {diq }}\right)$ also in the Faddeev equation (3.36).

For the numerical solution of this integral equation we discretize the integration variable $\tilde{\Omega}_{j}=j \Delta \tilde{\Omega} ; j=0, \ldots, N$, yielding the kernel as an $N \times N$ matrix. We then generalize the matrix equation to the eigenvalue equation

\footnotetext{
${ }^{4}$ In the following we suppress all diquark indices, e.g. $a_{\alpha, \mu}(i \Omega, i \tilde{\Omega})=a_{\mu}(i \Omega, i \tilde{\Omega})$.

${ }^{5}$ A similar variable substitution was implemented by Ishii et. al [27].

${ }^{6} \mathrm{~A}$ similar approach was employed for the solution of the Bethe-Salpeter equation for the diquarks 30].
} 


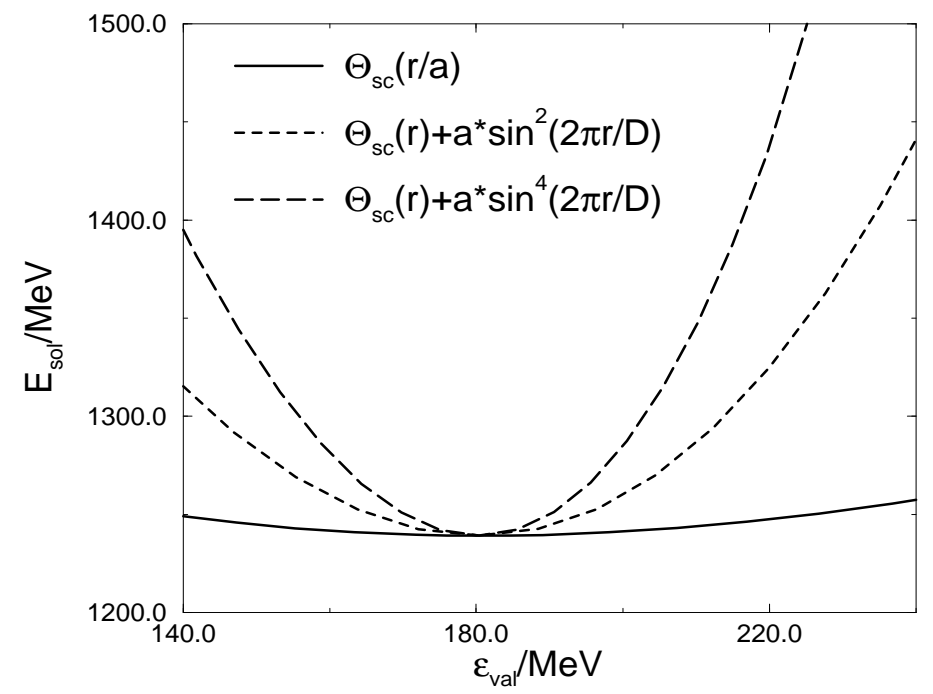

FIG. 2. Dependence of the soliton energy from the valence quark energy for three different parameterizations of the soliton profile. Results are for $m=450 \mathrm{MeV}$.

$$
\begin{aligned}
& {\left[\frac{1}{2}\left(i \Omega+\epsilon_{v a l}-2 \epsilon_{\nu}-\omega_{d i q}\right)-i \tilde{\Omega}_{j}\right]\left[\left(\tilde{\Omega}_{j}+\frac{i}{2}\left\{\epsilon_{v a l}-\omega_{d i q}-i \Omega\right\}\right)^{2}+\omega_{d i q}^{2}\right] a_{\nu}\left(i \Omega, i \tilde{\Omega}_{j}\right)} \\
& \quad+\frac{1}{4} \sum_{l, \kappa} \Delta \tilde{\Omega} \frac{\left\langle\nu\left|\Gamma_{d i q}\right| \kappa\right\rangle\left\langle\kappa\left|\Gamma_{d i q}\right| \mu\right\rangle}{i\left(\tilde{\Omega}_{j}+\tilde{\Omega}_{l}\right)+\omega_{d i q}+\epsilon_{\kappa}-\epsilon_{v a l}} a_{\mu}\left(i \Omega, i \tilde{\Omega}_{j}\right)=\lambda_{j}(i \Omega) a_{\nu}\left(i \Omega, i \tilde{\Omega}_{j}\right) .
\end{aligned}
$$

A solution of the Faddeev equation is given for a vanishing eigenvalue $\lambda_{j}\left(i \Omega_{B}\right)=0$ with the energy $\Omega_{B}$. Within this formulation there exists only one positive energy solution with $\Omega_{B}<\epsilon_{v a l}+\omega_{d i q}$. Possible negative solutions will again be rejected. If we replace $\Gamma_{d i q}$ in (3.37) with $\chi \Gamma_{d i q}$ we can explore the dependence of the solution on the coupling between quark and diquark. In this manner we observe that $\Omega_{B}$ approaches the free solution $\epsilon_{v a l}+\omega_{\text {diq }}$ for decreasing $\chi$. This justifies the above described procedure.

\section{NUMERICAL RESULTS AND DISCUSSION}

In the first step we calculate] the chiral angle $\Theta_{s c}(r)$ self-consistently in a box with radius $D$ by extremizing the energy functional (3.13). In order to later estimate the influence of the quark-quark and the diquark-quark bound states on the soliton we introduce a dimensionless parameter $a$, which measures the extension of the soliton profile, via $\Theta(r)=\Theta_{s c}(r / a)$. This choice is motivated by the softness of the scaling mode, i.e. a

\footnotetext{
${ }^{7} \mathrm{~A}$ complete description of the numerical treatment is given in [41].
} 


\begin{tabular}{lccc}
\hline \hline$a$ & $\omega_{\text {diq }} / \mathrm{MeV}$ & $2 \epsilon_{\text {val }}$ & $B_{q q} / \mathrm{MeV}$ \\
\hline 0.0 & 759 & 934 & 175 \\
0.2 & 761 & 934 & 173 \\
0.4 & 758 & 929 & 171 \\
0.6 & 672 & 808 & 136 \\
0.8 & 465 & 560 & 95 \\
1.0 & 295 & 360 & 65 \\
1.2 & 161 & 208 & 47 \\
\hline \hline
\end{tabular}

TABLE I. The energy $\omega_{d i q}$ of the diquark in the soliton, the energy $2 \epsilon_{v a l}$ of two uncorrelated quarks and the binding energy of the diquark $B_{q q}=2 \epsilon_{v a l}-\omega_{d i q}$ in dependence on the scaling parameter $a$ for constituent quark mass $m=450 \mathrm{MeV}$ and $g_{2}=g_{1}$.

weak dependence of the total soliton energy on the parameter $a$ in comparison to other variables. The energy of the valence quark, on the other hand, depends sensitively on a. As shown in Fig. 2 the soliton energy for the scaling mode is almost constant in the displayed range. For comparison the dependencies for two alternative parameterizations are presented. Both profiles are chosen such that they not only fulfill the usual boundary conditions but also the derivatives at the boundaries are unchanged.

In the next step we solve the Bethe-Salpeter equation (3.17) for the diquark fields?. In Tab. I we display the solution of the Bethe-Salpeter equation for different soliton profiles. The important result is that the diquarks are bound $\left(\omega_{\text {diq }}<2 \epsilon_{v a l}\right)$ for all considered profiles. Furthermore, the binding energy $B_{q q}=2 \epsilon_{v a l}-\omega_{d i q}$ decreases with growing soliton size. For a small soliton extension $(a<0.4)$ the diquark energy is almost independent of the soliton. Note, that the limit $a \rightarrow 0$ corresponds to a diquark in an empty box. On the contrary, for larger soliton extension $(a>0.5)$ we observe a strong dependence on the scaling factor $a$. This behavior of the diquark energy is inherited from the one of the valence quark energy. This indicates that the properties of the diquark are essentially determined by the valence quarks.

A further interesting point is the increasing diquark binding energy with growing constituent quark mass when the self-consistent soliton is employed as can be seen from

\footnotetext{
${ }^{8} \mathrm{~A}$ test of the numerical treatment in the finite box is given in appendix $\mathrm{A}$.
} 


\begin{tabular}{cccc}
\hline \hline$m / \mathrm{MeV}$ & $\omega_{\text {diq }} / \mathrm{MeV}$ & $2 \epsilon_{\text {val }}$ & $B_{q q} / \mathrm{MeV}$ \\
\hline 350 & 455 & 495 & 40 \\
400 & 368 & 421 & 53 \\
450 & 295 & 360 & 65 \\
500 & 225 & 303 & 78 \\
600 & 80 & 189 & 109 \\
\hline \hline
\end{tabular}

TABLE II. The same as Tab. in dependence on the constituent quark mass $m$ for the self-consistent soliton and $g_{2}=g_{1}$.

Tab. [1]. This feature is particularly astonishing because the valence quark energy decreases simultaneously.

These results for the diquark serve as input to the solution of the Faddeev equation (3.37). In Fig. 3 we display the resulting energy $\Omega_{B}$ as a function of the scaling variable $a$ for a constituent quark mass $m=450 \mathrm{MeV}$. For comparison, the energy of three uncorrelated valence quarks and the energy of a free diquark-quark pair are also shown. Again, in the entire region the diquark and quark are bound, although only weakly. In Tab. III we compare the quark-quark $\left(B_{q q}=2 \epsilon_{v a l}-\omega_{d i q}\right)$ and diquark-quark $\left(B_{q d}=\epsilon_{v a l}+\omega_{d i q}-\Omega_{B}\right)$ binding energies to the total binding energy

$$
B_{t o t}=3 \epsilon_{v a l}-\Omega_{B}=B_{q q}+B_{q d}
$$

for a diquark coupling constant $g_{2}=g_{1}$. We notice that the binding energy is carried to a large extent by the quark-quark correlations. While these have their maximal value for a vanishing soliton $(a \rightarrow 0)$ the residual diquark-quark interaction develops a maximum for moderate soliton extension. More distinct is the situation (Tab. [V]) for a diquark coupling constant twice as large, i.e. $g_{2}=2 g_{1}$. In particular, the total binding energy is strongly dominated by the diquark binding and is maximal for a vanishing soliton.

In the past some authors have used instantaneous or static approximations to solve the integral equation [22,24 26,42]. The instantaneous approximation corresponds to a quark exchange with vanishing relative energy $\tilde{\Omega}=0$ in eq. (3.37). Then the different quark channels decouple and the Faddeev equation simplifies to a purely algebraic equation:

$$
\left[i \Omega-\epsilon_{v a l}-\omega_{d i q}\right]\left[\left(\epsilon_{v a l}-\omega_{d i q}-i \Omega\right)^{2}-4 \omega_{d i q}^{2}\right]-2 \sum_{\kappa} \frac{\left\langle v a l\left|\Gamma_{d i q}\right| \kappa\right\rangle\left\langle\kappa\left|\Gamma_{d i q}\right| v a l\right\rangle}{\omega_{d i q}+\epsilon_{\kappa}-\epsilon_{v a l}}=0 .
$$

In the static approximation in addition the sum over the exchanged quark is restricted to the valence quark: 


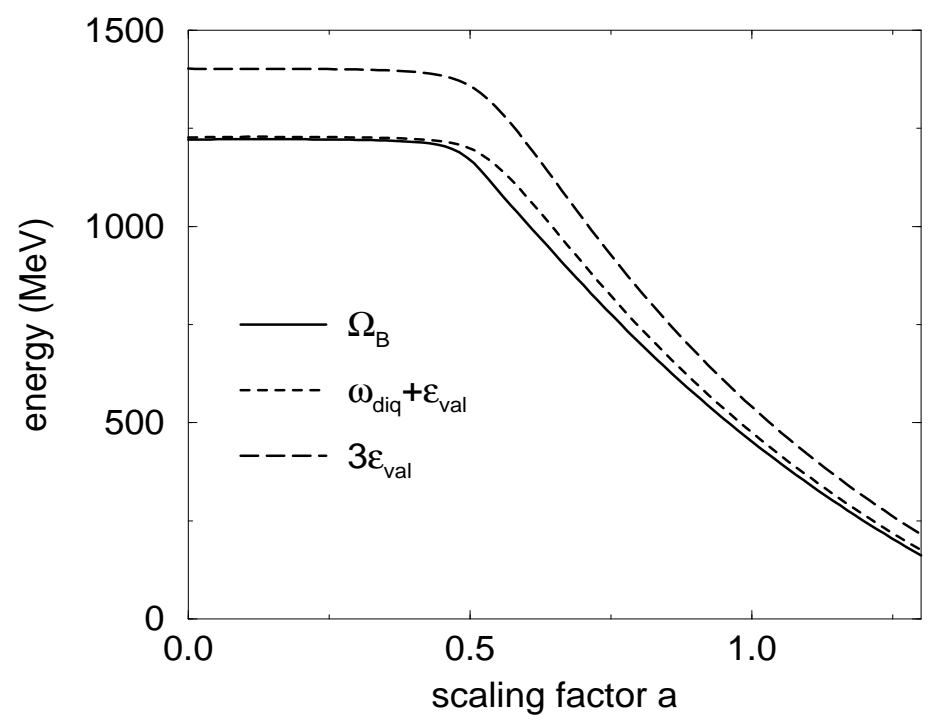

FIG. 3. Solution of the Faddeev equation (solid line), the energy of a free diquark-quark pair (short dashed line) and the energy of three uncorrelated valence quarks (long dash line) in dependence on the scaling factor $a$ for a constituent quark mass $m=450 \mathrm{MeV}$ and $g_{2}=g_{1}$.

\begin{tabular}{lcccc}
\hline \hline$a$ & $\Omega_{B} / \mathrm{MeV}$ & $B_{q q} / \mathrm{MeV}$ & $B_{q d} / \mathrm{MeV}$ & $B_{\text {tot }} / \mathrm{MeV}$ \\
\hline 0.0 & 1221 & 175 & 6 & 181 \\
0.2 & 1222 & 173 & 6 & 179 \\
0.4 & 1215 & 171 & 8 & 179 \\
0.6 & 1010 & 136 & 66 & 202 \\
0.8 & 705 & 95 & 40 & 135 \\
1.0 & 452 & 65 & 24 & 89 \\
1.2 & 249 & 47 & 15 & 62 \\
\hline \hline
\end{tabular}

TABLE III. The solution $\Omega_{B}$ of the Faddeev equation as well as the quark-quark $\left(B_{q q}\right)$ and diquark-quark binding energy $B_{q d}$ and the total binding energy $B_{t o t}$ of the three valence quarks for a constituent quark mass $m=450 \mathrm{MeV}$ and $g_{2}=g_{1}$. 


\begin{tabular}{lcccc}
\hline \hline$a$ & $\Omega_{B} / \mathrm{MeV}$ & $B_{q q} / \mathrm{MeV}$ & $B_{q d} / \mathrm{MeV}$ & $B_{\text {tot }} / \mathrm{MeV}$ \\
\hline 0.0 & 952 & 433 & 16 & 449 \\
0.2 & 957 & 428 & 17 & 445 \\
0.4 & 949 & 401 & 43 & 444 \\
0.6 & 787 & 315 & 110 & 425 \\
0.8 & 547 & 169 & 124 & 293 \\
1.0 & 374 & 108 & 59 & 167 \\
1.2 & 249 & 75 & 36 & 111 \\
\hline \hline
\end{tabular}

TABLE IV. The same as Tab. ПII for the coupling constant $g_{2}=2 g_{1}$.

$$
\left[i \Omega-\epsilon_{v a l}-\omega_{d i q}\right]\left[\left(\epsilon_{v a l}-\omega_{d i q}-i \Omega\right)^{2}-4 \omega_{d i q}^{2}\right]-2 \frac{\left\langle v a l\left|\Gamma_{d i q}\right| v a l\right\rangle\left\langle v a l\left|\Gamma_{d i q}\right| v a l\right\rangle}{\omega_{d i q}}=0 .
$$

The presence of the energy denominator indicates that these approximations should be well suited for large quark and diquark masses. In fact, Tab. $\mathrm{V}$ confirms this assessment. For a vanishing soliton the error is one per cent. When the soliton extension grows the valence quark as well as the diquark energy become smaller and therefore the error rises to about 20\%. This emphasizes the importance of a complete solution of the Faddeev equation for a solitonic background. In addition, this table shows only a small difference between both approximations. In comparison to the complete solution of the Faddeev equation both treatments possess an enhanced binding energy. Hence we conclude that retardation effects in the full calculation have a repulsive character. Both approximations also elucidate the importance of the above described variable substitution, because the free solution $i \Omega=\omega_{d i q}+\epsilon_{v a l}$ is obvious for vanishing diquark-quark coupling $\left(\Gamma_{d i q} \rightarrow 0\right)$. If we improperly had carried out the static approximation in equation (3.36) we would have had two unphysical solutions $i \Omega=2 \epsilon_{\text {val }}$ and $i \Omega= \pm \omega_{\text {diq }}$.

\section{A HYBRID MODEL FOR BARYONS}

Given the results of the last section we now consider a soliton configuration with correlated valence quarks. For that purpose we replace the contribution $3 \epsilon_{v a l}$ of the three uncorrelated valence quarks in the energy functional (3.13) by the solution of the Faddeev equation. The total energy of this configuration is given by

$$
E_{\text {sol }}^{(B)}=E_{\text {sea }}+\Omega_{B}=E_{\text {sol }}-B_{\text {tot }}<E_{\text {sol }}\left(=E_{\text {sea }}+3 \epsilon_{\text {val }}\right)
$$




\begin{tabular}{lccc}
\hline \hline$a$ & $\Omega_{B} / \mathrm{MeV}$ & $\Omega_{B}^{(\text {stat })} / \mathrm{MeV}$ & $\Omega_{B}^{(\text {inst })} / \mathrm{MeV}$ \\
\hline 0.0 & 1221 & 1202 & 1201 \\
0.2 & 1222 & 1204 & 1202 \\
0.4 & 1215 & 1194 & 1189 \\
0.6 & 1010 & 935 & 924 \\
0.8 & 705 & 630 & 628 \\
1.0 & 452 & 394 & 393 \\
1.2 & 249 & 203 & 202 \\
\hline \hline
\end{tabular}

TABLE V. The Solution $\Omega_{B}$ of the Faddeev equation (3.37) as well as the solution of the static approximation $\Omega_{B}^{(s t a t)}(4.3)$ and the instantaneous approximation $\Omega_{B}^{(i n s t)}(4.2)$ in dependence of the scaling factor $a$.

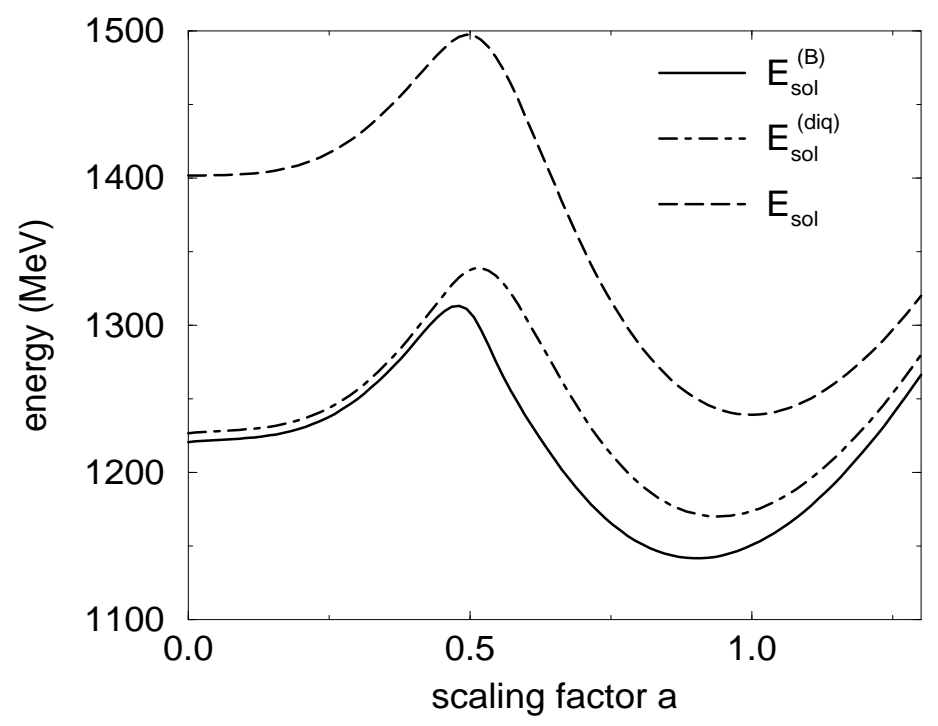

FIG. 4. The energy of the soliton with correlated valence quarks (solid line), the energy of the additive diquark-quark-soliton model (dash-dotted line) and the energy of the soliton with uncorrelated valence quarks (long dashed line) in dependence of the scaling factor $a$ for a constituent quark mass $450 \mathrm{MeV}$. 


\begin{tabular}{lccccc}
\hline \hline$m(\mathrm{MeV})$ & 350 & 400 & 450 & 500 & 600 \\
\hline$E_{\text {sol }}^{B}(\mathrm{MeV})$ & 1057 & 1140 & 1142 & 1108 & 1011 \\
$E_{\text {sea }}(\mathrm{MeV})$ & 0 & 0 & 570 & 569 & 606 \\
$\Omega_{B}(\mathrm{MeV})$ & 1057 & 1140 & 572 & 539 & 405 \\
$a$ & 0 & 0 & 0.90 & 0.85 & 0.80 \\
$\omega_{\text {diq }}(\mathrm{MeV})$ & 688 & 725 & 375 & 356 & 283 \\
$\sqrt{\left\langle r_{I=0}^{2}\right\rangle}$ & - & - & 0.56 & 0.53 & 0.47 \\
$g_{A}$ & 0.32 & 0.32 & 0.70 & 0.68 & 0.64 \\
\hline \hline
\end{tabular}

TABLE VI. The total energy of the lowest energy configuration $E_{\text {sol }}^{B}$ as well as the contribution from the Dirac sea $E_{\text {sea }}$ and from the correlated valence quarks $\Omega_{B}$ for $g_{2}=g_{1}$. Furthermore displayed is the scaling parameter $a$, the effective energy $\omega_{d i q}$ of the diquark, the isoscalar radius $\left\langle r_{I=0}^{2}\right\rangle^{1 / 2}$ and the axial coupling constant $g_{A}$.

and is shown in Fig. 1 as a function of the scaling parameter $a$ for the constituent quark mass of $450 \mathrm{MeV}$. For comparison, the energy of the pure soliton (3.13) $E_{\text {sol }}=E_{\text {sea }}+3 \epsilon_{\text {val }}$ and the energy of the additive diquark-quark soliton model $E_{\text {sol }}^{(d i q)}=E_{\text {sea }}+\omega_{\text {diq }}+\epsilon_{\text {val }}$ defined in [30] are also shown. As compared to the additive diquark-quark soliton picture the soliton with correlated valence quarks has a lower energy in the entire range shown. The minimum of this curve defines the hybrid model. Two other points in this figure also have a special meaning: First, the point $a=0$ on the same curve marks the model where a baryon is described as a bound state of a quark and a diquark with the meson fields fixed at their vacuum expectation values [25,27]. Note, that this configuration only corresponds to a local minimum of the energy. The other important point is the minimum of $E_{\text {sol }}$ at $a=1$ which is nothing else than the self-consistent soliton solution without any valence quark correlations [20]. Also the energy of this configuration is larger than the energy of the hybrid model.

In Table VI we display results for the lowest energy configuration for different constituent quark masses. This table shows that for a large enough constituent quark mass the hybrid soliton in fact is the minimal energy configuration. The lower limit of stability for the hybrid soliton is around $m \approx 430 \mathrm{MeV}$. Below this value the energy of the baryon $E_{\text {sol }}^{(B)}=\Omega_{B}$ is carried completely by the valence quarks. Herein, the diquark possesses an effective energy of about $700 \mathrm{MeV}$. In comparison, for $m \approx 450 \mathrm{MeV}$ the total energy of the 


\begin{tabular}{llllll}
\hline \hline$a$ & $g_{A}^{\text {val }}$ & $g_{A}^{\text {Fad }}$ & $g_{A}^{\text {diq }}$ & $g_{A}^{\text {sea }}$ & $g_{A}$ \\
\hline 0.0 & 0.325 & 0.325 & 0 & 0 & 0.325 \\
0.2 & 0.325 & 0.325 & 0.001 & -0.001 & 0.325 \\
0.4 & 0.324 & 0.323 & 0.017 & -0.012 & 0.328 \\
0.6 & 0.273 & 0.253 & 0.478 & -0.033 & 0.698 \\
0.8 & 0.246 & 0.239 & 0.485 & -0.032 & 0.692 \\
0.9 & 0.240 & 0.236 & 0.483 & -0.018 & 0.701 \\
1.0 & 0.237 & 0.234 & 0.483 & 0.005 & 0.722 \\
1.1 & 0.235 & 0.233 & 0.485 & 0.035 & 0.753 \\
1.2 & 0.234 & 0.232 & 0.489 & 0.074 & 0.795 \\
\hline \hline
\end{tabular}

TABLE VII. Contributions to the axial coupling constant $g_{A}$ in dependence of the scaling factor a. For comparison also the contribution $g_{A}^{v a l}$ of an uncorrelated valence quark is displayed.

self-consistent soliton configuration is composed half by the valence quarks and half by the polarized sea. The effective diquark energy drops down to the range $280-380 \mathrm{MeV}$.

In Table VI we also present results for the isoscalar radius $\left\langle r_{I=0}^{2}\right\rangle^{1 / 2}$ and the axial coupling constant $g_{A}$. These calculations need some comments: The isoscalar radius is calculated as the spatial expectation value

$$
\left\langle r_{I=0}^{2}\right\rangle=\int d^{3} \boldsymbol{r} r^{2} \rho(\boldsymbol{r})=4 \pi \int_{0}^{D} d r r^{4} \rho(r) .
$$

This expression is only well-defined for localized baryon densities. For non-localized baryon densities the radius depends on the box size $D$ which is the case when the soliton is absent $\square$. For the constituent quark mass $m=450 \mathrm{MeV}$ the isoscalar radius in the hybrid model $(0.56 \mathrm{fm})$ and the pure soliton model $(0.54 \mathrm{fm})$ are almost identical. We conclude from this slight increase that the sea is less attractive in the hybrid model despite of the stronger binding of the valence quarks. Note that both predictions are close to the experimental value $\left\langle r_{I=0}^{2}\right\rangle^{1 / 2}=0.62 \mathrm{fm}$.

\footnotetext{
${ }^{9}$ In the appendix $\mathrm{C}$ we give a full description of the calculation of static observables in the hybrid model.

${ }^{10}$ Because of this volume divergence we state nothing in this case.
} 


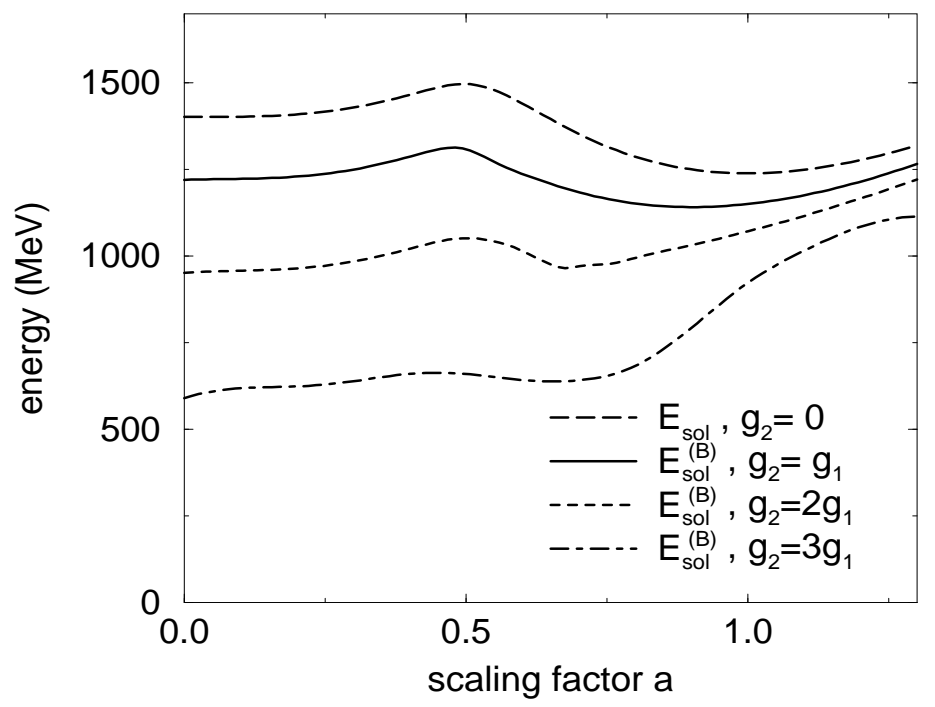

FIG. 5. The energy of the soliton with correlated valence quarks for different diquark coupling constants for a constituent quark mass $450 \mathrm{MeV}$.

More interesting are the results for the axial coupling constant

$$
g_{A}=g_{A}^{F a d}+g_{A}^{d i q}+g_{A}^{s e a}
$$

consisting of contributions from the sea part $g_{A}^{\text {sea }}$, the diquark $g_{A}^{\text {diq }}$ and the residual bound valence quark $g_{A}^{F a d}(c f$. appendix C). These contributions are collected in Tab. VII as a function of the scaling factor $a$. For comparison we also display the contribution $g_{A}^{v a l}$ of an uncorrelated valence quark. First, we observe only a small difference between a free and a correlated valence quark. As a function of the scaling factor we find two distinct regions: For small $a$ (no soliton) we have $g_{A}^{F a d} \approx 0.33$, for larger $a$ (soliton region) the value decreases to $g_{A}^{F a d} \approx 0.24$. On the other hand, the diquark contribution shows the opposite behavior. It vanishes in the case of a trivial background but it increases to more than twice the value of a single valence quark in the presence of the soliton. Finally, in both regions only small corrections occur, which originate from the sea contribution. The vanishing diquark contribution for $a=0$ also explains the small values for $m=350 \mathrm{MeV}$ and $m=400 \mathrm{MeV}$ in Tab. V1. In addition, these results indicate a missing component to the axial coupling constant: axial diquarks should also be included before comparing $g_{A}$ with the experimental value $g_{A}=1.26$ 43.

When we increase the diquark coupling $g_{2}$ we observe that stronger diquark correlations counteract the formation of a soliton. As can be seen from Fig. 5 the meson configuration, which minimizes $E_{\text {sol }}^{(B)}$, is shrinking with increasing $g_{2}$. Starting at a critical coupling a path is open to a vanishing soliton. The displayed curves correspond to four special cases. The pure soliton is given for $g_{2}=0$, whereas the second one $\left(g_{2}=g_{1}\right)$ 
describes the Fierz-symmetric case. In the case $g_{2}=2 g_{1}$ the energy $E_{\text {sol }}^{(B)}$ is identical to the physical nucleon mass [22] in the range where no soliton is present $(a<0.4)$. Finally, in the last case $g_{2}=3 g_{1}$ we have an additional symmetry - the Pauli-Gürsey symmetry 44 46] - giving the diquarks the same mass as the pions. However, this symmetry cannot be realized in nature since otherwise the baryon masses should be much smaller.

\section{CONCLUSIONS AND OUTLOOK}

In this paper we have constructed a hybrid model for the description of baryons within the framework of the NJL model. Using functional integral techniques we have converted the pure quark model into an effective theory of mesons, diquarks and baryons. As a first step we have solved the Bethe-Salpeter equation for an $S$-wave scalar diquark in the background of a soliton configuration. We have found that this diquark is kinematically stable against the decay into the two lightest quarks even in case the masses of the latter are strongly reduced by their interaction with the chiral soliton. In the next step we have integrated out the diquark fields, thereby inducing an interaction between quarks and the bound diquark through quark exchange. Using the ladder approximation for this exchange interaction a Faddeev type of equation has been derived, which includes the solitonic background field. This equation yields a three-quark bound state in the solitonic background. Using this result the hybrid has been constructed combining the three-quark bound state with the polarized sea. The energy of this hybrid is (for constituent quark masses $m>430 \mathrm{MeV}$ ) smaller than either the energy of the soliton with three uncorrelated quarks or the energy of the three-quark bound state with no soliton present. Hence the formation of such a hybrid is dynamically preferred as compared to either picture alone. In addition, the soliton size of the hybrid is much smaller than the size of the soliton with uncorrelated quarks, and the Faddeev amplitude, i.e. the baryon wave function in terms of quarks, significantly deviates from the case without soliton. These results show that baryons are presumably very much like hybrids containing both, solitonic meson clouds and three-quark correlations, and that the implementation of both features is crucial for a proper description of baryons. Unfortunately, the question whether a baryon is dominated by the valence quark structure or the soliton is still an open question. E. g. if one includes for the soliton also (axial-) vector mesons the baryon current is completely carried by the polarized Dirac sea [47,48]. To clarify the situation one should in addition include at the mean field level static diquark fields. The solution of such an improved soliton calculation would show whether there is still room for valence quark degrees of freedom beside vector meson degrees of freedom or whether they would exclude each other.

Within the hybrid model we have calculated the isoscalar radius $\left\langle r_{I=0}^{2}\right\rangle^{1 / 2}$ as well as 
the axial coupling constant $g_{A}$. While the isoscalar radius shows reasonable values in comparison with experiment the inclusion of axial diquarks seems to be important for the axial coupling constant. In order to calculate other observables one has to perform the last step in the construction of the hybrid model for baryons: one has to project the soliton solution to good spin and flavor states.

In this work we have considered the NJL model which is well suited for low-energy properties of hadrons. Nevertheless, for energies higher than twice the constituent quark mass, threshold effects associated with the unphysical decay into free quarks disturb the calculation. Therefore, a generalization to bilocal chiral models seems necessary in order to effectively incorporate quark confinement. Within these models there can also exist confined diquarks 49] on the one hand. On the other hand, soliton calculations have shown that inside a soliton propagating quarks [50 52] also can exist. Thus, the hybrid model introduced in this work presents a well suited starting point for further generalizations which appear necessary to deepen our understanding of the structure of baryons.

\section{ACKNOWLEDGMENTS}

The authors are grateful to L. Gamberg for carefully reading the manuscript. This work was partly supported by COSY under contract 41315266 and by Deutsche Forschungsgemeinschaft (DFG) under contract Re 856/2-2.

\section{APPENDIX A: TEST OF THE NUMERICAL TREATMENT IN THE FINITE BOX}

In order to test the numerical treatment in the finite box we compare the solution of (3.17) for a vanishing chiral angle $\Theta(r)=0$ with the solution in the continuum. The mass of the diquark is obtained from the Bethe-Salpeter equation [53]

$$
\left[-p^{2} A\left(p^{2}\right)+2 m^{2} B-\frac{m_{\pi}^{2} f_{\pi}^{2}}{6 m_{0} m g_{2} / g_{1}}\right]_{p^{2}=-m_{d i q}^{2}}=0
$$

with

$$
\begin{aligned}
A\left(p^{2}\right) & =\frac{1}{16 \pi^{2}} \int_{0}^{1} d \alpha \Gamma\left(0, \frac{m^{2}+\alpha(1-\alpha) p^{2}}{\Lambda^{2}}\right), \\
B & =\frac{1}{16 \pi^{2}} \Gamma\left(-1, \frac{m^{2}}{\Lambda^{2}}\right) .
\end{aligned}
$$

When we compare the solutions of the Bethe-Salpeter equations in the continuum (A1) and in the spherical box (3.17) we observe a relatively large difference (cf. the 


\begin{tabular}{ccccc}
\hline \hline$g_{2} / g_{1}$ & $m_{\text {diq }} / \mathrm{MeV}$ & $\omega_{\text {diq }} / \mathrm{MeV}$ & $\omega_{\text {diq }}^{(1)} / \mathrm{MeV}$ & $\omega_{d i q}^{(2)} / \mathrm{MeV}$ \\
\hline 0.0 & 900 & 934 & 934 & 909 \\
0.5 & 884 & 904 & 919 & 893 \\
1.0 & 736 & 759 & 778 & 747 \\
1.5 & 595 & 625 & 646 & 608 \\
2.0 & 463 & 502 & 527 & 480 \\
2.5 & 326 & 380 & 412 & 350 \\
3.0 & 140 & 241 & 288 & 188 \\
\hline \hline
\end{tabular}

TABLE VIII. The mass $m_{d i q}$ of a diquark in the continuum, the energy $\omega_{d i q}$ of a diquark as the solution of the Bethe-Salpeter equation in a spherical box, $\omega_{d i q}^{(1)}$ and $\omega_{d i q}^{(2)}$ from the approximation of eq. (A3) and eq. (A4) respectively in dependence of $g_{2} / g_{1}$.

$2^{\text {nd }}$ and $3^{\text {rd }}$ column in Tab. VIII). However, we have to take into account that in a finite box the quarks always possess finite momenta and therefore an increased energy $\epsilon_{\mu}= \pm \sqrt{m^{2}+(\mu \pi / D)^{2}}$. Now, we can consider two limits: First, in the limit of vanishing diquark coupling $\left(g_{2} \rightarrow 0\right)$ a diquark consists of two free quarks $\left(\omega_{d i q}=2 \epsilon_{v a l}\right)$. From this we obtain the approximation

$$
\omega_{d i q}^{(1)}=\sqrt{m_{d i q}^{2}+4(\pi / D)^{2}}
$$

for the energy of a diquark in a spherical box. Second, we consider the limit of a large quark-quark coupling. In this case, the diquark is a highly correlated state which can be viewed as an independent particle, hence

$$
\omega_{d i q}^{(2)}=\sqrt{m_{d i q}^{2}+(\pi / D)^{2}} .
$$

As can be seen from Tab. VIII and from Fig. 6 the diquark energy always lies within the two boundaries (A3) and ( $\mathrm{A} 4)$. In fact, in the limit of vanishing diquark coupling $\left(g_{2} \rightarrow 0\right)$ the energy of the diquark $\omega_{d i q}$ is very well approximated by $\omega_{d i q}^{(1)}$. The difference at large coupling constants between $\omega_{d i q}$ and $\omega_{\text {diq }}^{(2)}$ appears because of numerical effects at the boundary where the wave functions do not vanish completely. In the context of the hybrid model we consider bound states in the solitonic background. In this case, the wave functions are localized at the origin and the boundary effects are negligible. 


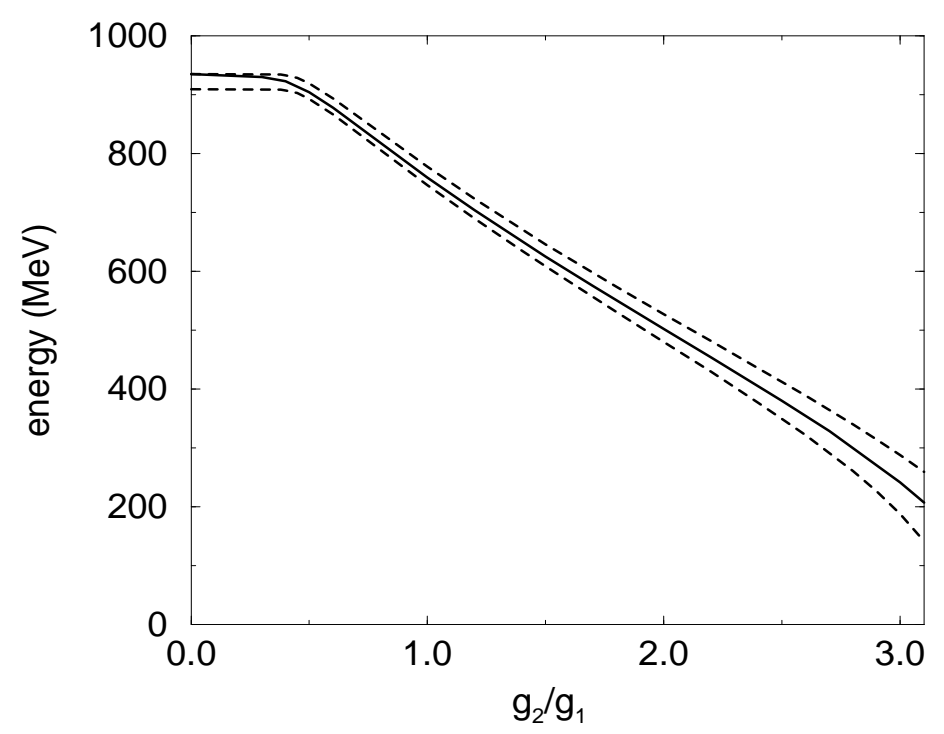

FIG. 6. The energy of the diquark in a spherical box (solid line) in dependence of $g_{2} / g_{1}$, the dashed lines corresponds to the upper $\left(\omega_{d i q}^{(1)}\right)$ and lower $\left(\omega_{d i q}^{(2)}\right)$ limit of the energy of the diquark.

\section{APPENDIX B: THE BARYON PROPAGATOR}

The baryon propagator can be evaluated by comparison of eq. (2.19) and (2.12)

$$
\frac{1}{2} \overline{\boldsymbol{\Psi}}^{\alpha}\left(\mathbf{G}_{B}^{v a l}\right)_{\alpha \beta} \boldsymbol{\Psi}^{\beta}=\frac{1}{2} \overline{\boldsymbol{\xi}} \mathbf{G} \boldsymbol{\xi} .
$$

Using the definitions of $\boldsymbol{\xi}$ and $\overline{\boldsymbol{\xi}}(2.13)$ we can write

$$
\left(\mathbf{G}_{B}^{v a l}\right)_{\alpha \beta}=\left(\begin{array}{cc}
\Delta_{\alpha} G_{11} \Delta_{\beta}^{*} & -\Delta_{\alpha} G_{12} \Delta_{\beta} \\
-\Delta_{\alpha}^{*} G_{21} \Delta_{\beta}^{*} & \Delta_{\alpha}^{*} G_{22} \Delta_{\beta}
\end{array}\right),
$$

where we have used the inversion of the quark propagator

$$
\mathbf{G}_{0}=\left(\begin{array}{ll}
G_{11} & G_{12} \\
G_{21} & G_{22}
\end{array}\right)
$$

which is obtained from eq. (2.14) by neglecting the baryon sources on the diagonal elements. The elements of this matrix are given by

$$
\begin{aligned}
& G_{11}=\left(1+\mathcal{G}_{0} \Delta \tilde{\mathcal{G}_{0}} \tilde{\Delta}\right)^{-1} \mathcal{G}_{0} \\
& G_{12}=-\mathcal{G}_{0} \Delta\left(1+\tilde{\mathcal{G}_{0}} \tilde{\Delta} \mathcal{G}_{0} \Delta\right)^{-1} \tilde{\mathcal{G}_{0}} V \\
& G_{21}=-V^{-1} \tilde{\mathcal{G}}_{0}^{-1} \tilde{\Delta}\left(1+\mathcal{G}_{0} \Delta \tilde{\mathcal{G}_{0}} \tilde{\Delta}\right)^{-1} \mathcal{G}_{0}, \\
& G_{22}=-V^{-1}\left(1+\tilde{\mathcal{G}_{0}} \tilde{\Delta} \mathcal{G}_{0} \Delta\right)^{-1} \tilde{\mathcal{G}_{0}} V .
\end{aligned}
$$




\section{APPENDIX C: CALCULATION OF STATIC OBSERVABLES}

The calculation of observables in the hybrid model separates in a valence quark part and a sea part:

$$
\langle\hat{O}\rangle=\langle\hat{O}\rangle_{v a l}+\langle\hat{O}\rangle_{s e a}
$$

Because of the ansatz for the baryon wave function (3.33)

$$
|B\rangle=\sum_{\alpha} \sum_{\nu}^{\left(\epsilon_{\nu}>0\right)} a_{\alpha \nu}|\alpha, \nu\rangle
$$

with

$$
B(x, y)=\langle x, y \mid B\rangle=\int d \omega d E \sum_{\alpha} \sum_{\nu}^{\left(\epsilon_{\nu}>0\right)} a_{\alpha \nu}(E, \omega) B_{\alpha \nu}^{0}\left(\vec{x}, x_{4} ; \vec{y}, y_{4}\right)
$$

we can in addition separate the valence quark part in a diquark and a residual valence quark part:

$$
\begin{aligned}
\langle\hat{O}\rangle_{v a l} & =\langle B|\hat{O}| B\rangle_{v a l} \\
& =\sum_{\alpha, \beta} \sum_{\nu, \mu}^{\left(\epsilon_{\nu, \mu}>0\right)} a_{\alpha \nu}^{*} a_{\beta \mu}\langle\alpha, \nu|\hat{O}| \beta, \mu\rangle \\
& =\sum_{\alpha, \beta} \sum_{\nu}^{\left(\epsilon_{\nu}>0\right)} a_{\alpha \nu}^{*} a_{\beta \nu}\langle\alpha|\hat{O}| \beta\rangle+\sum_{\alpha} \sum_{\nu, \mu}^{\left(\epsilon_{\nu, \mu}>0\right)} a_{\alpha \nu}^{*} a_{\alpha \mu}\langle\nu|\hat{O}| \mu\rangle \\
& =\langle\hat{O}\rangle_{\text {diq }}+\langle\hat{O}\rangle_{F a d} .
\end{aligned}
$$

To take into account the internal quark structure of the diquarks we use the form

$$
\begin{aligned}
O & =\langle\hat{O}\rangle=\int \mathcal{D} \mathbf{q} \mathcal{D} \overline{\mathbf{q}} q^{\dagger} \hat{O} q \exp \left(\frac{1}{2} \int \overline{\mathbf{q}} \mathbf{G}^{-1} \mathbf{q}\right) \\
& =\frac{1}{2} \int \mathcal{D} \mathbf{q} \mathcal{D} \overline{\mathbf{q}} \overline{\mathbf{q}} \hat{\mathbf{O}} \mathbf{q} \exp \left(\frac{1}{2} \int \overline{\mathbf{q}} \mathbf{G}^{-1} \mathbf{q}\right) \\
& =\left.\frac{\partial}{\partial \beta} \int \mathcal{D} \mathbf{q} \mathcal{D} \overline{\mathbf{q}} \exp \left(\frac{1}{2} \int \overline{\mathbf{q}}\left(\mathbf{G}^{-1}+\beta \hat{\mathbf{O}}\right) \mathbf{q}\right)\right|_{\beta=0} \\
& =\left.\frac{\partial}{\partial \beta} \operatorname{Tr} \log \left(\mathbf{G}^{-1}+\beta \hat{\mathbf{O}}\right)\right|_{\beta=0},
\end{aligned}
$$

where we have extended the operator $\hat{O}$ according to the Nambu-Gorkov formalism:

$$
\hat{\mathbf{O}}=\left(\begin{array}{cc}
\gamma_{0} \hat{O} & 0 \\
0 & -V \tilde{O} V^{\dagger}
\end{array}\right)
$$


with $\tilde{O}=V \hat{O}^{T} \gamma_{0} V^{-1}$. Expanding the expression (C5) in powers of the diquark field the zeroth order term corresponds to $\langle\nu|\hat{O}| \mu\rangle$ in the quark term whereas the second order term yields the diquark part $\langle\alpha|\hat{O}| \beta\rangle$ in eq. (C4).

The part of the residual valence quark is given by

$$
\langle\hat{O}\rangle_{F a d}=\int \frac{d \tilde{\Omega}}{2 \pi} \sum_{\alpha} \sum_{\nu, \mu}^{\left(\epsilon_{\nu, \mu}>0\right)} a_{\alpha \nu}^{*}\left(i \Omega_{B}, i \tilde{\Omega}\right) a_{\alpha \mu}\left(i \Omega_{B}, i \tilde{\Omega}\right)\langle\nu|\hat{O}| \mu\rangle
$$

The sea part is calculated as usual in the pure soliton model [20]:

$$
\langle\hat{O}\rangle_{\text {sea }}=-\frac{1}{2} \sum_{\nu}\langle\nu|\hat{O}| \nu\rangle_{\operatorname{sgn}}\left(\epsilon_{\nu}\right) \operatorname{erfc}\left(\left|\frac{\epsilon_{\nu}}{\Lambda}\right|\right)
$$

\section{Baryon number $\hat{B}$}

In the Nambu-Gorkov formalism the baryon number operator is defined as

$$
\hat{\mathbf{B}}=\left(\begin{array}{cc}
\gamma_{0} \hat{B} & 0 \\
0 & -V \gamma_{0} \hat{B} V^{\dagger}
\end{array}\right) \quad \text { with } \quad \hat{B}=\mathbb{1}_{C} / N_{C}=\mathbb{1}_{C} / 3
$$

The term of second order in the diquark field is expressed as

$$
\begin{gathered}
B_{d i q}=i \int \frac{d \omega}{2 \pi} d r^{\prime} r^{\prime 2} \Delta_{\alpha}^{*}\left(r^{\prime},-i \omega\right) \int d r r^{2} \Delta_{\alpha}(r, i \omega) \int \sum_{\nu \mu} \int d \Omega d \Omega^{\prime} R_{2}\left(\omega ; \epsilon_{\nu}, \epsilon_{\mu}\right) \\
*\left[\Psi_{\mu}^{\dagger}\left(\boldsymbol{r}^{\prime}\right) \Psi_{\nu}\left(\boldsymbol{r}^{\prime}\right) \Psi_{\nu}^{\dagger}(\boldsymbol{r}) \Psi_{\mu}(\boldsymbol{r})\right]
\end{gathered}
$$

with the regularization function

$$
\begin{aligned}
& R_{2}\left(i \omega ; \epsilon_{\nu}, \epsilon_{\mu}\right)=(\mathrm{C} 11) \\
& \frac{i \omega\left(\omega^{2}+\left(\epsilon_{\nu}-\epsilon_{\mu}\right)^{2}\right)}{4 \sqrt{\pi}} \int_{0}^{1} d \alpha \alpha^{2} \frac{\Gamma\left(\frac{5}{2},\left[\alpha \epsilon_{\mu}^{2}+(1-\alpha) \epsilon_{\nu}^{2}+\alpha(1-\alpha) \omega^{2} / \Lambda^{2}\right]\right)}{\left[\alpha \epsilon_{\mu}^{2}+(1-\alpha) \epsilon_{\nu}^{2}+\alpha(1-\alpha) \omega^{2}\right]^{5 / 2}} .
\end{aligned}
$$

\section{Baryon density}

For the calculation of the baryon densities we need the identity operator $\hat{O}=\mathbb{1}$ as well as a spatial dependent variation variable $\beta=\beta(\boldsymbol{r})$ in eq. (C5). The diquark contribution can be written as

$$
B_{d i q}(\boldsymbol{r})=\sum_{\nu \mu} \Psi_{\nu}^{\dagger}(\boldsymbol{r}) \Psi_{\mu}(\boldsymbol{r}) R_{3}\left(i \omega ; \epsilon_{\nu}, \epsilon_{\mu}\right)
$$


with the regularization function

$$
\begin{aligned}
R_{3}\left(i \omega ; \epsilon_{\nu}, \epsilon_{\mu}\right)= & \frac{1}{4 \sqrt{\pi}} \int_{0}^{1} d \alpha \sum_{\kappa}\left[\frac{\Gamma\left(\frac{3}{2},\left[\alpha \epsilon_{\mu}^{2}+(1-\alpha) \epsilon_{\kappa}^{2}+\alpha(1-\alpha) \omega^{2} / \Lambda^{2}\right]\right)}{\left[\alpha \epsilon_{\mu}^{2}+(1-\alpha) \epsilon_{\kappa}^{2}+\alpha(1-\alpha) \omega^{2}\right]^{3 / 2}}\right. \\
& \left.-\frac{\Gamma\left(\frac{3}{2},\left[\alpha \epsilon_{\nu}^{2}+(1-\alpha) \epsilon_{\kappa}^{2}+\alpha(1-\alpha) \omega^{2} / \Lambda^{2}\right]\right)}{\left[\alpha \epsilon_{\nu}^{2}+(1-\alpha) \epsilon_{\kappa}^{2}+\alpha(1-\alpha) \omega^{2}\right]^{3 / 2}}\right] \\
& \frac{\left[i \omega+\epsilon_{\kappa}-\epsilon_{\nu}\right]\left[-i \omega+\epsilon_{\kappa}-\epsilon_{\mu}\right]}{\epsilon_{\mu}-\epsilon_{\nu}}\left\langle\mu\left|\Gamma_{d i q}(i \omega)\right| \kappa\right\rangle\left\langle\kappa\left|\tilde{\Gamma}_{d i q}(-i \omega)\right| \nu\right\rangle \\
& \left(\epsilon_{\mu}+\epsilon_{\nu}\right) \frac{\Gamma\left(\frac{3}{2},\left[\alpha \epsilon_{\mu}^{2}+(1-\alpha) \epsilon_{\nu}^{2}+\alpha(1-\alpha) \omega^{2} / \Lambda^{2}\right]\right)}{\left[\alpha \epsilon_{\mu}^{2}+(1-\alpha) \epsilon_{\nu}^{2}+\alpha(1-\alpha) \omega^{2}\right]^{3 / 2}}\left\langle\mu\left|\Gamma_{d i q} \tilde{\Gamma}_{d i q}\right| \mu\right\rangle
\end{aligned}
$$

\section{Axial coupling constant}

Using the operator $\hat{O}=-\frac{1}{3} \sigma^{3} \tau^{3}$ [20] the axial coupling constant is given by

$$
g_{A}^{d i q}=-\frac{1}{3} \sum_{\nu, \mu}\left\langle\nu\left|\sigma^{3} \tau^{3}\right| \mu\right\rangle R_{3}\left(i \omega ; \epsilon_{\nu}, \epsilon_{\mu}\right)
$$

with the regularization function $R_{3}$ defined in (C13). Note, that eq.(C14) is only valid for grand spin eigenstates $|\mu\rangle$. 


\section{REFERENCES}

[1] G. 't Hooft, Nucl. Phys. B72, 461 (1974).

[2] E. Witten, Nucl. Phys. B156, 269 (1979).

[3] T. H. R. Skyrme, Proc. Roy. Soc. A260, 127 (1961).

[4] G. S. Adkins, C. R. Nappi, and E. Witten, Nucl. Phys. B228, 552 (1983).

[5] G. Holzwarth (Ed.), Baryons as Skyrme Solitons (World Scientific Publ. Comp., Singapore, 1993).

[6] M. Gell-Mann, Phys. Rev. 8, 214 (1964).

[7] G. Karl and E. Obryk, Nucl. Phys. B8, 609 (1968).

[8] D. Faiman and A. W. Hendry, Phys. Rev. 173, 1720 (1968).

[9] R. P. Feynman, M. Kislinger, and F. Ravndal, Phys. Rev. D3, 2706 (1971).

[10] J. Kogut and L. Susskind, Phys. Rev. D9, 697,3391 (1974).

[11] P. Hasenfratz and J. Kuti, Phys. Rep. 40, 75 (1978).

[12] D. B. Lichtenberg, Phys. Rev. 155, 1601 (1967).

[13] P. D. DeSouza and D. B. Lichtenberg, Phys. Rev. 161, 1513 (1967).

[14] A. Chodos and C. Thorn, Phys. Rev. D12, 2733 (1975).

[15] M. Rho, Phys. Rep. 240, 1 (1994).

[16] S. Nadkarni, H. B. Nielsen, and I. Zahed, Nucl. Phys. B253, 308 (1985).

[17] A. Hosaka, H. Toki, and W. Weise, Nucl. Phys. A506, 501 (1990).

[18] A. Hosaka and H. Toki, Phys. Lett. B322, 1 (1993).

[19] Y. Nambu and G. Jona-Lasinio, Phys. Rev. 122, 345 (1961).

[20] R. Alkofer, H. Reinhardt, and H. Weigel, Phys. Rep. 265, 139 (1996).

[21] D. Ebert and H. Reinhardt, Nucl. Phys. B271, 188 (1986).

[22] A. Buck, R. Alkofer, and H. Reinhardt, Phys. Lett. B286, 29 (1992).

[23] N. Ishii, W. Bentz, and K. Yazaki, Phys. Lett. B318, 26 (1993).

[24] S. Huang and J. Tjon, Phys. Rev. C49, 1702 (1994).

[25] H. Meyer, Phys. Lett. B337, 37 (1994).

[26] C. Hanhart and S. Krewald, Phys. Lett. B344, 55 (1995).

[27] N. Ishii, W. Bentz, and K. Yazaki, Nucl. Phys. A587, 617 (1995). 
[28] A. Buck and H. Reinhardt, Phys. Lett. B356, 168 (1995).

[29] H. Reinhardt, Phys. Lett. B244, 316 (1990).

[30] U. Zückert, R. Alkofer, H. Weigel, and H. Reinhardt, Phys. Lett. B362, 1 (1995).

[31] R. T. Cahill, Austr. J. Phys. 42, 171 (1989).

[32] D. Ebert and L. Kaschluhn, Phys. Lett. B297, 367 (1992).

[33] Y. Nambu, Phys. Rev. 117, 648 (1960).

[34] L. P. Gorkov, JETP 7, 993 (1958).

[35] J. R. Schrieffer, Theory of Superconductivity (Benjamin, New York, 1964).

[36] J. Schwinger, Phys. Rev. 82, 664 (1951).

[37] H. Weigel, R. Alkofer, and H. Reinhardt, Nucl. Phys. B387, 638 (1992).

[38] H. Reinhardt and R. Wünsch, Phys. Lett. B215, 577 (1988).

[39] T. Meissner, F. Grümmer, and K. Goeke, Phys. Lett. B227, 296 (1989).

[40] R. Alkofer, Phys. Lett. B236, 310 (1990).

[41] R. Alkofer and H. Weigel, Comp. Phys. Comm. 82, 30 (1994).

[42] V. Keiner, Z. Phys. A354, 87 (1996).

[43] Particle Data Group, Phys. Rev. D50, 1173 (1994).

[44] W. Pauli, Nuovo Cim. 6, 205 (1957).

[45] F. Gürsey, Nuovo Cim. 7, 411 (1958).

[46] U. Vogl and W. Weise, Prog. Nucl. Part. Phys. 27, 195 (1991).

[47] R. Alkofer, H. Reinhardt, H. Weigel, and U. Zückert, Phys. Rev. Lett. 69, 1874 (1992).

[48] U. Zückert, R. Alkofer, H. Weigel, and H. Reinhardt, Mod. Phys. Lett. A10, 67 (1995).

[49] A. Bender, C. D. Roberts, and L. v. Smekal, Phys. Lett. B380, 7 (1996).

[50] M. R. Frank, P. C. Tandy, and G. Fai, Phys. Rev. C43, 2808 (1991).

[51] P. C. Tandy and M. R. Frank, Aust. J. Phys. 44, 181 (1991).

[52] M. R. Frank and P. C. Tandy, Phys. Rev. C46, 338 (1992).

[53] C. Weiss, A. Buck, R. Alkofer, and H. Reinhardt, Phys. Lett. B312, 6 (1993). 